\title{
Universal Divergence Estimation for Finite-alphabet
}

\section{Sources}

\author{
Haixiao Cai Sanjeev R. Kulkarni Sergio Verdú \\ Dept. of Electrical Engineering \\ Princeton University \\ Princeton, NJ 08544
}

\begin{abstract}
We study universal estimation of divergence from the realizations of two unknown finite-alphabet sources. We present two algorithms using techniques from data compression. The first divergence estimator applies the Burrows-Wheeler block sorting transform to the concatenation of the two realizations; consistency of this estimator is shown for all finite memory sources. The second divergence estimator is based on the Context Tree Weighting method; consistency is shown for all sources whose memory length does not exceed a known bound. Experimental results show that both algorithms perform similarly and outperform string-matching and plug-in methods.
\end{abstract}

\section{Index Terms}

block sorting, Burrows-Wheeler Transform, Context Tree Weighting method, divergence estimation, information divergence, Markov sources, relative entropy.

\section{INTRODUCTION}

Although (Kullback-Leibler) divergence (also known as relative entropy) is a fundamental information measure, special cases of which are mutual information and entropy, the problem of divergence estimation of sources whose distributions are unknown has received relatively little attention.

The sources of interest are finite-alphabet, finite-memory Markov sources, denoted by $q_{z}$ and $p_{x}$. Denote the alphabet set by $\chi$, and the state set by $\mathcal{S}$. The input to the estimator consists of a realization of length $n$ from source $q_{z}$, denoted by $\mathbf{z}=z^{n}$, and a realization of length $n$ from source $p_{x}$, denoted by $\mathbf{x}=x^{n}$.

This work was supported in part by ARL MURI under Grant number DAAD19-00-1-0466, Draper Laboratory under IR\&D 6002 Grant DL-H-546263, and the National Science Foundation under Grant CCR-0312413. 
Ziv and Merhav [15] applied the idea of LZ parsing to divergence estimation. They developed an interesting scheme to estimate the divergence of two finite-alphabet, finite-order, stationary Markov processes. The LZ incremental parsing algorithm parses the sequence $\mathbf{z}$ of length $n$ into $c\left(z^{n}\right)$ distinct phrases such that each phrase is the shortest string which is not a previously parsed phrase. The entropy $H\left(q_{z}\right)$ can be estimated by $\frac{1}{n} c\left(z^{n}\right) \log _{2} c\left(z^{n}\right)$. Analogously, $\mathbf{z}$ is parsed into $c\left(z^{n} \| x^{n}\right)$ longest phrases, which appear in $\mathbf{x}$. The term $-\frac{1}{n} \log _{2} p_{x}\left(z^{n}\right)$ can be estimated by $\frac{1}{n} c\left(z^{n} \| x^{n}\right) \log _{2} n$. Then an estimator of the divergence rate $D\left(q_{z} \| p_{x}\right)$ is given by

$$
\frac{1}{n} c\left(z^{n} \| x^{n}\right) \log _{2} n-\frac{1}{n} c\left(z^{n}\right) \log _{2} c\left(z^{n}\right) .
$$

Consistency of this estimator is shown in [15] if the observations are generated by independent Markov sources, and is universal in the sense of not depending on the order $d$ or any other information about the transition probability matrices of the sources.

Another algorithm based on LZ compression was introduced in [1] and applied to problems motivated by linguistics. Unlike [15], the approach in [1] is heuristic and there is no claim that the algorithm converges to the divergence of the sources. The idea is to approximate the divergence $D\left(q_{z} \| p_{x}\right)$ by calculating the number of bits per character wasted to encode the sequence emitted by source $q_{z}$ with a source code that has been trained by a realization of source $p_{x}$. To that end, a realization from source $q_{z}$ is partitioned into a long sequence $\mathbf{z}$ and a shorter sequence $\mathbf{z}_{0}$, which is appended to a long sequence $\mathbf{x}$ from source $p_{x}$. The new sequence $\mathbf{x}+\mathbf{z}_{0}$ is compressed by gzip ${ }^{1}$ to $L_{\mathbf{x}+\mathbf{z}_{0}}$ bits, while $\mathbf{x}$ alone is compressed to $L_{\mathbf{x}}$ bits. The difference $\Delta_{\mathbf{x z}_{0}}=L_{\mathbf{x}+\mathbf{z}_{0}}-L_{\mathbf{x}}$ is the coding length of $\mathbf{z}_{0}$ using the coding trained by $\mathbf{x}$. Similarly, $\Delta_{\mathbf{z z}_{0}}=L_{\mathbf{z}+\mathbf{z}_{0}}-L_{\mathbf{z}}$. The divergence between $q_{z}$ and $p_{x}$ is approximated by

$$
\frac{\Delta_{\mathbf{x z}_{0}}-\Delta_{\mathbf{z z}_{0}}}{\left|\mathbf{z}_{0}\right|}
$$

where $\left|\mathbf{z}_{0}\right|$ is the number of characters of the short sequence $\mathbf{z}_{0}$. A new class of "normalized information distance" loosely based on the non-computable notion of Kolmogorov complexity is proposed in [11], and then applied to the genome phylogeny problem and the problem of building language trees similar to [1]. The method in [11] to approximate the measure therein is heuristic (see also the discussion in [10]).

In this paper, we present two divergence estimation algorithms, both of which are motivated by techniques in data compression. The first estimator, originally proposed in [3], uses the Burrows-Wheeler block sorting Transform (BWT) [2], while the second estimator uses the Context Tree Weighting method (CTW) [13]. We prove the convergence of our divergence estimators assuming that both sources are possibly

\footnotetext{
${ }^{1}$ A commercial embodiment of LZ data compression.
} 
dependent stationary ergodic Markov sources, a case for which the following almost-sure convergence result is known to hold [9]:

$$
\begin{aligned}
& D\left(q_{z} \| p_{x}\right) \\
& =\sum_{s \in \mathcal{S}} q_{z}(s) \sum_{\alpha \in \chi} q_{z}(\alpha \mid s) \log _{2}\left(\frac{q_{z}(\alpha \mid s)}{p_{x}(\alpha \mid s)}\right) \\
& =\lim _{n \rightarrow \infty} \frac{1}{n} \log _{2} \frac{q_{z}\left(Z^{n}\right)}{p_{x}\left(Z^{n}\right)} \quad \text { a.s. }
\end{aligned}
$$

A variety of compression algorithms have been proposed using the BWT as a front end followed by modules such as move-to-front, run-length coding, and adaptive Huffman coding. An entropy estimator based on the BWT was proposed in [4] using a uniform segmentation scheme. Based on that, we can show that segments of the BWT output sequence are close to an i.i.d. sequence. This property is exploited in our algorithm to estimate divergence without knowing the memory length of the sources.

Recently, experimental results have been reported [7] using the Context Tree Weighting method (CTW) [13] for classification of binary sequences. The similarity metric used in [7] for classification can be seen to be an estimate of $D\left(q_{z}|| p_{x}\right)+H\left(q_{z}\right)$.

Another natural method, which we refer to as the plug-in method, for the estimation of divergence between two finite-memory sources consists of assuming an upper bound on the memory length of the sources, computing the empirical conditional probabilities and stationary probabilities by counting the number of symbols following each state, and plugging the estimates in (1).

The rest of the paper is organized as follows. In Section II, we present the divergence estimator based on the BWT. Convergence results for this estimator are proved in Section III. The divergence estimator based on CTW is presented and analyzed in Section IV. Finally, experimental results on randomly generated sources and on text files (such as novels and the Bible) are presented in Section V. Experiments on randomly generated tree sources show the superiority of the BWT-based and CTW-based algorithms over the plug-in and LZ-based methods.

\section{DiVERGEnCE ESTIMATOR Via The BWT}

\section{A. The Burrows-Wheeler Transform (BWT)}

The Burrows-Wheeler Transform is a reversible block-sorting algorithm [2]. It operates on a sequence of $n$ symbols (with a unique "end-of-file” symbol '\$' appended), produces all $n$ cyclic shifts of the original sequence, sorts them lexicographically, and outputs the last column of the sorted table. For finite-memory 
sources, performing the BWT on a reversed data sequence groups together symbols in the same state. Using the BWT followed by segmentation is the basic idea behind the entropy estimation in [4]. However, how to extend this idea to divergence estimation is not immediately clear because the two sources may have different state sets. The transitions for the two sequences do not occur in the same places, and need not correspond to the same contexts. In order to overcome this hurdle, we next introduce the joint BWT of two sequences.

For our purposes, it is important to extend the BWT to operate on the concatenation of two sequences $\mathbf{x}$ and $\mathbf{z}$ defined on the same alphabet. We concatenate $\mathbf{x}$ and $\mathbf{z}$, adding ' $\$$ ' to the end of each sequence, then sort the table of cyclic shifts, and output the last column. The information of the origin of each symbol (whether from $\mathbf{x}$ or $\mathbf{z}$ ) is kept, but the sorting does not discriminate symbols by their origin. For clarity, we will use upper case letters to represent $\mathbf{x}$ and lower case letters to represent $\mathbf{z}$ in the following. It should always be understood that $a$ and $A$ designate the same symbol, with upper/lower case indicating which source the symbol originates from. For example, if $\mathbf{z}=$ "banana" $\mathrm{x}=$ "anbaba". then we feed "banana\$ANBABA\$" to the BWT, and the output is "Bbn\$nBN\$AaaAaA" as shown in Figure 1. Unlike the standard use of the BWT in data compression, here we do not need to recover the original

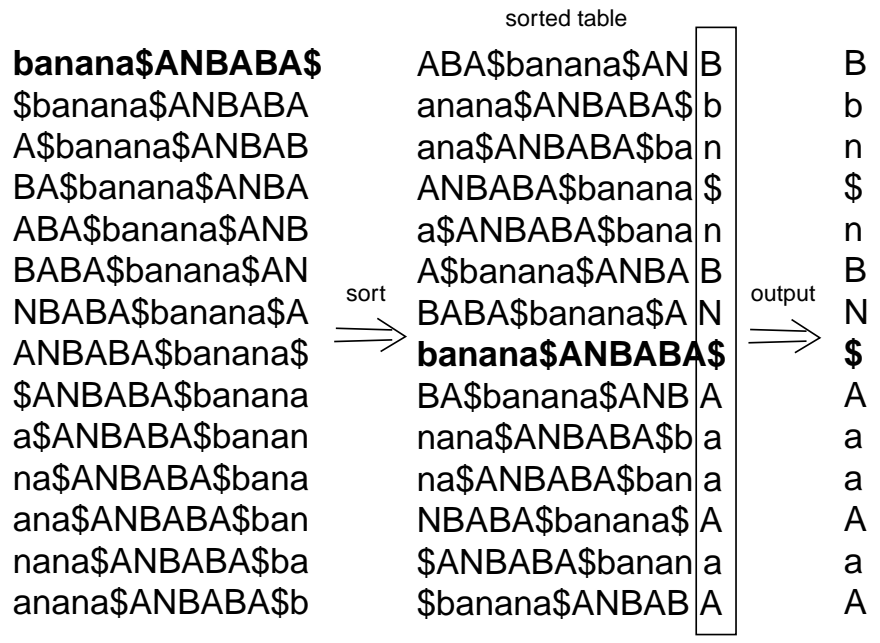

Fig. 1. An example of the joint BWT.

sequences from their block-sorted version.

\section{B. Divergence Estimator via the BWT}

A basic task of our divergence estimator is to estimate conditional empirical distributions, which can be done through segmentation of the BWT output. As we will see, the divergence estimator runs the joint BWT on the concatenation of the two sequences. 
For convenience, we will focus on the case in which the lengths of $\mathrm{x}$ and $\mathrm{z}$ are identical. However, it will be evident that this restriction is not necessary. In the discrete setting of this paper, it is convenient to decompose divergence as a difference:

$$
D\left(q_{z}|| p_{x}\right)=\lim _{n \rightarrow \infty}-\frac{1}{n} \log _{2} p_{x}\left(Z^{n}\right)-H\left(q_{z}\right)
$$

We can estimate the entropy appearing in the right side in a variety of ways; in particular we can use the BWT-based algorithm in [4]. Thus we focus on the estimation of the cross term in (3), i.e. on the sum of the entropy and divergence:

$$
S\left(q_{z} \| p_{x}\right) \triangleq D\left(q_{z} \| p_{x}\right)+H\left(q_{z}\right)=\lim _{n \rightarrow \infty}-\frac{1}{n} \log _{2} p_{x}\left(Z^{n}\right) \text { a.s. }
$$

Thus, once $H\left(q_{z}\right)$ has been estimated, the problem of estimating $D\left(q_{z}|| p_{x}\right)$ boils down to estimating $\frac{1}{n} \log _{2} p_{x}\left(z^{n}\right)$. To this end we will proceed as if we were estimating the entropy of $\mathbf{x}$, but instead of evaluating $\log _{2} p_{x}(\cdot)$ at $\mathbf{x}$, we will evaluate it at $\mathbf{z}$. The joint BWT allows us to do this in a natural way. We segment the joint BWT output based on statistics of the symbols from x. Ideally, this would segment the joint BWT output at the transition points of $\mathrm{x}$, resulting in piecewise i.i.d. segments for $\mathrm{x}$ according to the memory structure of the source $p_{x}$. In each segment, we compute the empirical probability of each symbol according to $\mathbf{x}$, which gives estimates of the conditional probabilities in each state of the source $p_{x}$. These are used to estimate the probability (according to $p_{x}$ ) of the observed symbols from $\mathbf{z}$.

Note that the BWT of $\mathbf{z}$ can be obtained by simply retaining the symbols of $\mathbf{z}$ from the joint BWT. Thus we actually need only perform the joint BWT to estimate both the entropy term $H\left(q_{z}\right)$ and the divergence $D\left(q_{z} \| p_{x}\right)$. However, to estimate $H\left(q_{z}\right)$ we segment the joint BWT output according to the symbols from $\mathbf{z}$, but to estimate $S\left(q_{z} \| p_{x}\right)$ we segment the output according to $\mathbf{x}$.

We run the BWT on the concatenation of the reversed sequence of $\mathbf{x}$ and $\mathbf{z}$ as explained in Section II-A. Our estimator for $S\left(q_{z} \| p_{x}\right)$ has the following steps (see Figure 2).

a) Run the joint BWT on the reversed concatenation of $\mathbf{z}$ and $\mathbf{x}$.

b) Segmentation according to symbols from $\mathrm{x}$. This will be described further in Section II-C. If a uniform segmentation strategy is adopted, the segments contain equal number of symbols from $\mathbf{x}$ but in general, different numbers of symbols from $\mathbf{z}$. Therefore, this segmentation induces a segmentation on the symbols of the sequence $\mathbf{z}$ which is different from the segmentation performed for the estimation of the entropy of $\mathbf{z}$.

c) Estimate the first order distribution (according to $\mathrm{x}$ ) within each segment as shown in Figure 3. Denote the number of occurrences of symbol $a$ (from $\mathbf{z}$ ) in the $j$ th segment (according to the 


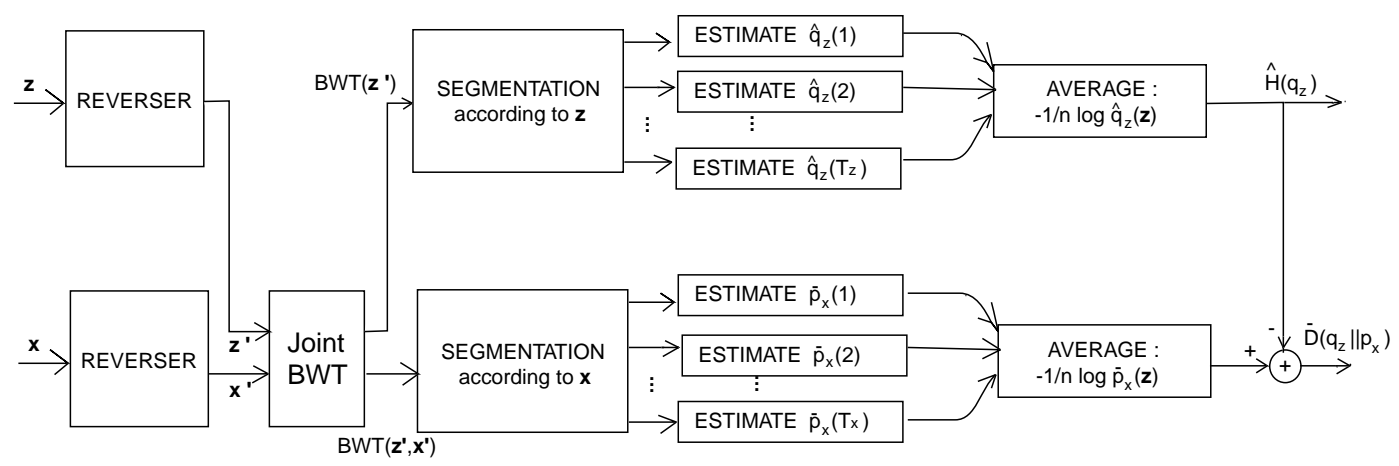

Fig. 2. Block diagram of the divergence estimator via the BWT.

segmentation with respect to x) by $\bar{N}_{j}(a)$. Similarly, $\bar{N}_{j}(A)$ denotes the number of occurrences of symbol $A$ (from $\mathbf{x})$ in the $j$ th segment.

An estimate of the probability of the symbols from $\mathrm{x}$ in the $j$ th segment is

$$
\bar{p}_{\mathbf{x}}(A, j)=\frac{\bar{N}_{j}(A)+\Delta}{\sum_{B \in \chi} \bar{N}_{j}(B)+|\chi| \Delta} .
$$

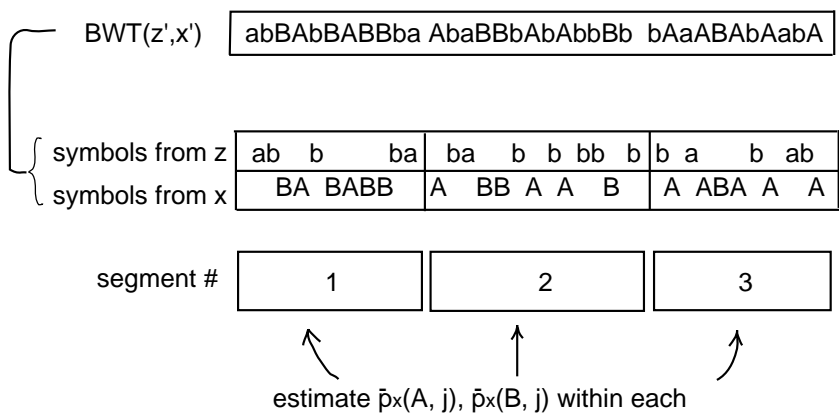

Fig. 3. The joint BWT, segmentation and estimation.

If a probability estimate in a segment is zero, the logarithm of the probability is $-\infty$ and the divergence estimate is $+\infty$. Therefore, a small bias $\Delta>0$ is introduced in the probability estimates in order to deal with this issue. In Section III, we will analyze the impact of this bias on our estimator.

The contribution of the $j$ th segment to the estimate of $\log _{2} \bar{p}_{\mathbf{x}}\left(z^{n}\right)$ is

$$
\log _{2} \bar{p}_{\mathbf{x}}(j)=\sum_{a \in \chi} \bar{N}_{j}(a) \log _{2} \bar{p}_{\mathbf{x}}(A, j) .
$$

Recall that $a$ and $A$ index the same symbol in the alphabet with the uppercase notation indicating that the symbol comes from $\mathrm{x}$ and the lowercase indicating that the symbol comes from $\mathrm{z}$. Hence, 
the term $\bar{N}_{j}(a)$ in (6) has " $a$ " since this is a count of the number of occurrences in $\mathbf{z}$, while the term $\log _{2} \bar{p}_{\mathbf{x}}(j, A)$ has " $A$ " since this is an estimate of the log probability of the symbol based on $\mathbf{x}$.

d) Average across segments. The estimate of the sought-after divergence-plus-entropy functional is obtained by averaging the contributions from the $T_{x}$ segments:

$$
\bar{S}\left(q_{z} \| p_{x}\right)=-\frac{1}{n} \sum_{j=1}^{T_{x}} \log _{2} \bar{p}_{\mathbf{x}}(j) .
$$

\section{Segmentation}

Segmentation is an important step in the divergence estimator. As discussed in [4], there are two natural approaches. One approach, called adaptive segmentation, is to detect the transitions based on the empirical distributions of the BWT output. The other, called uniform segmentation, simply divides the BWT output into segments so that each segment contains an equal number of symbols, denoted by $w(n)$, from the sequence according to which we are segmenting. For example, in Figure 3 each segment has the same number of symbols from x. However, the segments of the joint BWT output (containing symbols from both $\mathbf{x}$ and $\mathbf{z}$ ) may be different in length due to the different numbers of symbols from $\mathbf{z}$.

Recall that to estimate $H\left(q_{z}\right)$, the segmentation is performed according to symbols from $\mathbf{z}$ (indicated by the lower case symbols), while the divergence-plus-entropy functional used to estimate $D\left(q_{z} \| p_{x}\right)$ requires a segmentation according to symbols from $\mathrm{x}$ (indicated by the upper case symbols).

\section{Performance Analysis of the BWT-Based Divergence estimator}

In this section, we prove the convergence of our estimator with uniform segmentation and obtain conditions on the growth of segment length for our algorithm. We assume that the sources are stationary Markov with a finite unknown order. The state sequence forms a Markov chain, which is assumed to be irreducible and aperiodic with a unique stationary distribution. The set of states for both sources is $\mathcal{S}=\left\{s_{1}, s_{2}, \ldots, s_{|\mathcal{S}|}\right\}$, which is in lexicographical order, as sorted by the BWT. Denote by $S_{i} \in \mathcal{S}$ the state from which symbol $z_{i}$ emanates, where $1 \leq i \leq n$. We further assume that the conditional probabilities $p_{x}(a \mid s)$ and $q_{z}(a \mid s)$ satisfy $p_{x}(a \mid s)<1$ and $q_{z}(a \mid s)<1$, for all $a \in \chi$ and $s \in \mathcal{S}$.

\section{A. Empirical probabilities in each segment}

In order to prove convergence of the divergence estimator, we must show that the empirical probabilities in almost every $w(n)$-segment are close to the true conditional probabilities for a corresponding state of 
the Markov source (Lemma 1), and there are not too many symbols from $\mathbf{z}$ falling into any single segment (Lemma 3). The variability of the number of symbols from $\mathbf{z}$ falling in each segment presents a major challenge.

Let $N_{\mathbf{x}}(s)$ be the number of occurrences of state $s$ in $\mathbf{x}$. For $1 \leq k \leq n / w(n)$, let $S_{n}(k)$ be the state of segment $k$ if all symbols in segment $k$ emanate from the same state. We have

$$
S_{n}(k)=s_{i}
$$

if both

$$
k w(n) \leq \sum_{1 \leq j \leq i} N_{\mathbf{x}}\left(s_{j}\right)
$$

and

$$
(k-1) w(n) \geq \sum_{j<i} N_{\mathbf{x}}\left(s_{j}\right)
$$

are satisfied for some $1 \leq i \leq|\mathcal{S}|$; if there is no such $i$, then $S_{n}(k)=\phi$, which means it is a "bad" segment containing a state transition. Let $B=\left\{j: 1 \leq j \leq n / w(n), \quad S_{n}(j)=\phi\right\}$ be the set of bad segments containing a state transition. Denote by $\hat{p}_{\mathbf{x}}(a, k)$ the empirical probability of symbol $a$ in the $k$-th segment. Let $K_{s}=\left|\left\{j: 1 \leq j \leq n / w(n), \quad S_{n}(j)=s\right\}\right|$ be the number of segments whose state is $s \in S$. Let us define the following quantities:

$$
\overline{\bar{p}}_{\mathbf{x}}(a \mid s)=\frac{1}{K_{s}} \sum_{j: S_{n}(j)=s} \hat{p}_{\mathbf{x}}(a, j),
$$

and

$$
\overline{\bar{p}}_{\mathbf{x}}(s)=\frac{w(n) K_{s}}{n}
$$

$\overline{\bar{p}}_{\mathbf{x}}(a \mid s)$ is an estimate of the conditional probability $p_{x}(a \mid s)$, and $\overline{\bar{p}}_{\mathbf{x}}(s)$ is an estimate of the stationary probability $\mu_{x}(s)$. The proof of Theorems 1 and 2 in [4] introduces a fictitious estimator, that discards bad segments and fuses segments corresponding to the same states. In fact, $\overline{\bar{p}}_{\mathbf{x}}(a \mid s)$ and $\overline{\bar{p}}_{\mathbf{x}}(s)$ are estimates in the fused segment corresponding to state $s$ as in the fictitious entropy estimator.

Lemma 1 If we choose the segment length $w(n)=n^{\frac{1}{2}+\eta}$, where $0<\eta<1 / 2$, then for any $a \in \chi$ and $s \in \mathcal{S}$, we have

$$
E\left[\frac{1}{K_{s}} \sum_{j: S_{n}(j)=s}\left(\hat{p}_{\mathbf{x}}(a, j)-p_{x}(a \mid s)\right)^{2}\right]=O\left(\frac{\log n}{n^{\frac{1}{2}-\eta}}\right)
$$

and therefore

$$
E\left[\frac{w(n)}{n} \sum_{k \notin B} \sum_{a \in \chi}\left(\hat{p}_{\mathbf{x}}(a, k)-p_{x}\left(a \mid S_{n}(k)\right)\right)^{2}\right]=O\left(\frac{\log n}{n^{\frac{1}{2}-\eta}}\right),
$$


where the expectation is with respect to the sequence $\mathbf{x}$.

Proof: By the convergence rate of the entropy estimator for finite state Markov sources [4, Theorem 2], we have

$$
\begin{aligned}
& E\left[\left(\frac{w(n)}{n} \sum_{k \notin B} \sum_{a \in \chi} \hat{p}_{\mathbf{x}}(a, k) \log \frac{1}{\hat{p}_{\mathbf{x}}(a, k)}-H\left(p_{x}\right)\right)^{2}\right] \\
& =O\left(\frac{w^{2}(n) \log ^{2} n}{n^{2}}\right)+O\left(\frac{\log ^{2} n}{w^{2}(n)}\right) \\
& =O\left(\frac{\log ^{2} n}{n^{1-2 \eta}}\right)
\end{aligned}
$$

and

$$
\begin{aligned}
& E\left[\left(\sum_{s \in S} \overline{\bar{p}}_{\mathbf{x}}(s) \sum_{a \in \chi} \overline{\bar{p}}_{\mathbf{x}}(a \mid s) \log \frac{1}{\overline{\bar{p}}_{\mathbf{x}}(a \mid s)}-H\left(p_{x}\right)\right)^{2}\right] \\
& =O\left(\frac{\log ^{2} n}{n^{1-2 \eta}}\right) .
\end{aligned}
$$

It follows that, for any $a \in \chi$ and any $s \in \mathcal{S}$,

$$
\begin{aligned}
& E\left[\left(\overline{\bar{p}}_{\mathbf{x}}(a \mid s) \log \frac{1}{\overline{\bar{p}}_{\mathbf{x}}(a \mid s)}-\frac{1}{K_{s}} \sum_{j: S_{n}(j)=s} \hat{p}_{\mathbf{x}}(a, j) \log \frac{1}{\hat{p}_{\mathbf{x}}(a, j)}\right)^{2}\right] \\
& =O\left(\frac{\log ^{2} n}{n^{1-2 \eta}}\right),
\end{aligned}
$$

when $w(n)=n^{\frac{1}{2}+\eta}$, and $0<\eta<1 / 2$. Then (13) follows from (17), using Lemma 2 and the fact that

$$
E\left[\left(\overline{\bar{p}}_{\mathbf{x}}(a \mid s)-p_{x}(a \mid s)\right)^{2}\right]=O\left(\frac{1}{n^{1-2 \eta}}\right) .
$$

In Lemma 2, we will drop $a, s$ and the subscript $\mathbf{x}$ in $\overline{\bar{p}}_{\mathbf{x}}(a \mid s)$ and $\hat{p}_{\mathbf{x}}(a, j)$ and simply use notation $\hat{p}(j)$ for probabilities, where $1 \leq j \leq K$.

\section{Lemma 2 Let}

$$
E=\overline{\bar{p}} \log \frac{1}{\overline{\bar{p}}}-\frac{1}{K} \sum_{1 \leq j \leq K} \hat{p}(j) \log \frac{1}{\hat{p}(j)}
$$

where

$$
\overline{\bar{p}}=\frac{1}{K} \sum_{1 \leq j \leq K} \hat{p}(j) .
$$

There exists $\delta>0$, such that if $\{\hat{p}(j)\}$ satisfies $E<\delta$, then

$$
\frac{1}{K} \sum_{1 \leq j \leq K}(\hat{p}(j)-\overline{\bar{p}})^{2} \leq 2 E
$$


Proof: We want to find the maximum

$$
F(\overline{\bar{p}}, E, K) \triangleq \max _{\hat{p}(\cdot)}\left(\frac{1}{K} \sum_{j}(\hat{p}(j)-\overline{\bar{p}})^{2}\right),
$$

under the constraints that

$$
\begin{gathered}
\overline{\bar{p}} \log \frac{1}{\overline{\bar{p}}}-\left(\frac{1}{K} \sum_{j} \hat{p}(j) \log \frac{1}{\hat{p}(j)}\right)=E \\
\frac{1}{K} \sum_{j} \hat{p}(j)=\overline{\bar{p}}
\end{gathered}
$$

and for $1 \leq j \leq K$,

$$
0 \leq \hat{p}(j) \leq 1
$$

We first consider maximizing over only three of the variables $\hat{p}(\cdot)$ holding the rest fixed and arbitrary. It is equivalent to maximize $p_{1}^{2}+p_{2}^{2}+p_{3}^{2}$ under the constraints that $0 \leq p_{1} \leq 1,0 \leq p_{2} \leq 1,0 \leq p_{3} \leq 1$,

$$
\frac{p_{1}+p_{2}+p_{3}}{3}=p
$$

and

$$
\frac{p_{1} \log \frac{1}{p_{1}}+p_{2} \log \frac{1}{p_{2}}+p_{3} \log \frac{1}{p_{3}}}{3}=p \log \frac{1}{p}-\varsigma
$$

where $0<p<1$ and $\varsigma>0$ are constants. Without loss of generality, we assume $1 \geq p_{1} \geq p_{2} \geq p_{3} \geq 0$. It is shown in Appendix A that the maximum is achieved when either $p_{1}=1$ or $p_{1}>p_{2}=p_{3}$.

Going back to the original constrained maximization problem (22), we see that the maximizing $\hat{p}(j)$ 's should take at most three distinct values, one of them is 1 . We have $F(\overline{\bar{p}}, E, K) \leq G_{3}(\overline{\bar{p}}, E)$, where

$$
G_{3}(\overline{\bar{p}}, E) \triangleq \max _{p_{2}, p_{3}, \alpha_{2}, \alpha_{3}}\left\{\left(1-\alpha_{2}-\alpha_{3}\right)+\alpha_{2} p_{2}^{2}+\alpha_{3} p_{3}^{2}-\overline{\bar{p}}^{2}\right\}
$$

under the constraints

$$
\begin{gathered}
\left(1-\alpha_{2}-\alpha_{3}\right)+\alpha_{2} p_{2}+\alpha_{3} p_{3}=\overline{\bar{p}} \\
-\alpha_{2} p_{2} \log p_{2}-\alpha_{3} p_{3} \log p_{3}=-\overline{\bar{p}} \log \overline{\bar{p}}-E
\end{gathered}
$$

and $\alpha_{2} \geq 0, \alpha_{3} \geq 0,1-\alpha_{2}-\alpha_{3} \geq 0$, and $1 \geq p_{2} \geq 0,1 \geq p_{3} \geq 0$. It is shown in Appendix $\mathrm{B}$ that the maximum of $G_{3}(\overline{\bar{p}}, E)$ is achieved when $p_{2}=1$ (or equivalently $\alpha_{2}=0$ ). Therefore

$$
G_{3}(\overline{\bar{p}}, E)=\left(1-\alpha_{3}\right)+\alpha_{3} p_{3}^{2}-\overline{\bar{p}}^{2}
$$

where

$$
\left(1-\alpha_{3}\right)+\alpha_{3} p_{3}=\overline{\bar{p}}
$$


and

$$
-\alpha_{3} p_{3} \log p_{3}=-\overline{\bar{p}} \log \overline{\bar{p}}-E
$$

As $E$ goes to $0, p_{3}$ goes to $\overline{\bar{p}}$, and $G_{3}(\overline{\bar{p}}, E)=\left(\overline{\bar{p}}-p_{3}\right)(1-\overline{\bar{p}})$ goes to zero. We have

$$
\lim _{E \rightarrow 0} \frac{G_{3}(\overline{\bar{p}}, E)}{E}=\frac{(1-\overline{\bar{p}})^{2}}{-\log \overline{\bar{p}}+\overline{\bar{p}}-1},
$$

which belongs to $(0,2)$. This completes the proof.

\section{B. Divergence estimator with uniform segmentation for Markov sources}

Theorem 1 Let $\mathbf{z}$ and $\mathrm{x}$ be sequences of length $n$ generated from finite-alphabet finite-state Markov sources $q_{z}$ and $p_{x}$ respectively. Assume that $D\left(q_{z}|| p_{x}\right)<+\infty$, and $q_{z}(a \mid s)<1, p_{x}(a \mid s)<1$ for any $a \in \chi$ and $s \in \mathcal{S}$. Let $\bar{D}_{n}\left(q_{z} \| p_{x}\right)$ denote the divergence estimate using uniform segmentation with segment length $w(n)=n^{\frac{1}{2}+\eta}$, where $0<\eta<1 / 2$. Then $\bar{D}_{n}\left(q_{z} \| p_{x}\right)$ converges to $D\left(q_{z} \| p_{x}\right)$ in probability.

Proof: We first decompose the divergence into two terms, and estimate each separately.

$$
\begin{gathered}
D\left(q_{z} \| p_{x}\right)=S\left(q_{z} \| p_{x}\right)-H\left(q_{z}\right) . \\
\bar{D}_{n}\left(q_{z} \| p_{x}\right)=\bar{S}_{n}\left(q_{z} \| p_{x}\right)-\hat{H}_{n}\left(q_{z}\right) .
\end{gathered}
$$

Since we have proved the convergence of the entropy estimator in [4], it remains to prove that the cross term $\bar{S}_{n}\left(q_{z}|| p_{x}\right) \rightarrow S\left(q_{z} \| p_{x}\right)$ in probability.

Let $N_{\mathbf{z}}(s)$ be the number of occurrences of state $s$ in $\mathbf{z}$, and $N_{\mathbf{z}}(a, s)$ be the number of occurrences of symbol $a$ emanating from state $s$ in $\mathbf{z}$. Let $N_{\mathbf{z}}(a, k)$ be the number of occurrences of symbol $a$ from $\mathbf{z}$ in the $k$-th segment, and $N_{\mathbf{z}}(k)$ be the total number of symbols from $\mathbf{z}$ in the $k$-th segment. Note $w(n)$ is the number of symbols from $\mathbf{x}$ in each segment, so $N_{\mathbf{z}}(k)$ and $w(n)$ are different. Define

$$
\check{S}_{n}\left(q_{z}|| p_{x}\right) \triangleq-\frac{1}{n} \sum_{s \in \mathcal{S}} \sum_{a \in \chi} N_{\mathbf{z}}(a, s) \log _{2} p_{x}(a \mid s) .
$$

In Appendix D, we prove that

$$
E\left[\left(\check{S}_{n}\left(q_{z} \| p_{x}\right)-S\left(q_{z} \| p_{x}\right)\right)^{2}\right]=O\left(\frac{1}{n}\right)
$$

Now consider the estimator

$$
\bar{S}_{n}\left(q_{z} \| p_{x}\right)=-\frac{1}{n} \sum_{k}\left(\sum_{a \in \chi} N_{\mathbf{z}}(a, k) \log _{2} \bar{p}_{\mathbf{x}}(a, k)\right),
$$

where $\bar{p}_{\mathbf{x}}(a, k)$ is the estimate of $p_{x}(a \mid \cdot)$ in the $k$-th segment. We only need to prove that

$$
\bar{S}_{n}\left(q_{z}|| p_{x}\right)-\check{S}_{n}\left(q_{z}|| p_{x}\right) \rightarrow 0
$$


in probability, as $n \rightarrow \infty$. In fact, $\breve{S}_{n}\left(q_{z}|| p_{x}\right)=-\frac{1}{n} \log p_{x}\left(z^{n}\right)$, where $p_{x}$ is the probability of the source generating $\mathbf{x}$. And $\bar{S}_{n}\left(q_{z} \| p_{x}\right)=-\frac{1}{n} \log \bar{p}_{\mathbf{x}}\left(z^{n}\right)$, where $\bar{p}_{\mathbf{x}}$ is an estimate of $p_{x}$.

$$
\bar{p}_{\mathbf{x}}\left(z^{n}\right)=\prod_{i=1}^{n} \bar{p}_{\mathbf{x}}\left(z_{i}, \ell(\mathbf{x}, \mathbf{z}, i)\right)
$$

where $\ell(\mathbf{x}, \mathbf{z}, i)$ is the index of the segment that $z_{i}$ falls into after the joint BWT of $\mathbf{x}$ and $\mathbf{z}$.

Let

$$
\tau \triangleq \min _{a \in \chi s \in \mathcal{S}}\left\{p_{x}(a \mid s): p_{x}(a \mid s)>0\right\} .
$$

For any $\epsilon>0$, there exists $\delta_{\epsilon}>0$, such that $\left|\log \bar{p}_{\mathbf{x}}-\log p_{x}\right| \leq \epsilon$, for any $\left|\bar{p}_{\mathbf{x}}-p_{x}\right| \leq \delta_{\epsilon}$ and $p_{x} \geq \tau$. We proceed to bound the number of $z_{i}$ that falls into segments that do not have good estimates.

We use the biased estimates $\bar{p}_{\mathbf{x}}(a, j)$ instead of the empirical estimates $\hat{p}_{\mathbf{x}}(a, j)$, so that probability estimates are lower bounded by $\frac{\Delta}{w(n)+\Delta|\chi|}$,

$$
\log \frac{1}{\bar{p}_{\mathbf{x}}(a, j)} \leq \log \left(\frac{w(n)+\Delta|\chi|}{\Delta}\right)=O(\log n) .
$$

By Lemma 1 , for any $a \in \chi$ and $s \in \mathcal{S}$ we have

$$
E\left[\frac{w(n)}{n} \sum_{j: S_{n}(j)=s}\left(\hat{p}_{\mathbf{x}}(a, j)-p_{x}(a \mid s)\right)^{2}\right]=O\left(\frac{\log n}{n^{\frac{1}{2}-\eta}}\right) .
$$

Since $\left|\bar{p}_{\mathbf{x}}(a, j)-\hat{p}_{\mathbf{x}}(a, j)\right| \leq \frac{(|\chi|-1) \Delta}{w(n)}, \bar{p}_{\mathbf{x}}(a, j)$ have the same property as $\hat{p}_{\mathbf{x}}(a, j)$, namely,

$$
E\left[\frac{1}{n^{\frac{1}{2}-\eta}} \sum_{j: S_{n}(j)=s}\left(\bar{p}_{\mathbf{x}}(a, j)-p_{x}(a \mid s)\right)^{2}\right]=O\left(\frac{\log n}{n^{\frac{1}{2}-\eta}}\right),
$$

for any $a \in \chi$ and $s \in \mathcal{S}$.

Let $A_{n, a, s}(\delta)$ and $E_{n, a, s}(\delta)$ be the sets of segment indices.

$$
\begin{aligned}
& A_{n, a, s}(\delta)=\left\{k: 1 \leq k \leq n / w(n), S_{n}(k)=s, \text { and }\left|\bar{p}_{\mathbf{x}}(a, k)-p_{x}(a \mid s)\right|>\delta\right\} . \\
& E_{n, a, s}(\delta)=\left\{k: 1 \leq k \leq n / w(n), S_{n}(k)=s, \text { and }\left|\bar{p}_{\mathbf{x}}(a, k)-p_{x}(a \mid s)\right| \leq \delta\right\} .
\end{aligned}
$$

It follows from (45) that, for $\delta=\delta_{\epsilon}>0$,

$$
\operatorname{Pr}\left\{\left|A_{n, a, s}(\delta)\right|>\frac{\log ^{2} n}{\delta^{2}}\right\} \leq \frac{C}{\log n} .
$$


Now let us write

$$
\begin{aligned}
& \left|\bar{S}_{n}\left(q_{z}|| p_{x}\right)-\check{S}_{n}\left(q_{z}|| p_{x}\right)\right| \\
& =\sum_{i=1}^{n} \frac{1}{n}\left|\log p_{x}\left(z_{i} \mid S_{i}\right)-\log \bar{p}_{\mathbf{x}}\left(z_{i}, \ell(\mathbf{x}, \mathbf{z}, i)\right)\right| \\
& =\sum_{a \in \chi} \sum_{s \in \mathcal{S}} \sum_{i: z_{i}=a, S_{i}=s} \frac{1}{n}\left|\log p_{x}\left(z_{i} \mid S_{i}\right)-\log \bar{p}_{\mathbf{x}}\left(z_{i}, \ell(\mathbf{x}, \mathbf{z}, i)\right)\right| \\
& \triangleq \sum_{a \in \chi} \sum_{s \in \mathcal{S}} J(a, s) .
\end{aligned}
$$

Under the assumption $D\left(q_{z}|| p_{x}\right)<+\infty$, if $p_{x}(a \mid s)=0, q_{z}(a \mid s)=0$, and therefore $J(a, s)=0$. If $p_{x}(a \mid s) \geq \tau>0$, we have

$$
\begin{aligned}
& J(a, s) \\
\leq & \sum_{i: z_{i}=a, \ell(\mathbf{x}, \mathbf{z}, i) \in E_{n, a, s}(\delta)} \frac{1}{n}\left|\log p_{x}\left(z_{i} \mid S_{i}\right)-\log \bar{p}_{\mathbf{x}}\left(z_{i}, \ell(\mathbf{x}, \mathbf{z}, i)\right)\right| \\
& +\sum_{i: z_{i}=a, \ell(\mathbf{x}, \mathbf{z}, i) \in A_{n, a, s}(\delta)} \frac{1}{n}\left|\log p_{x}\left(z_{i} \mid S_{i}\right)-\log \bar{p}_{\mathbf{x}}\left(z_{i}, \ell(\mathbf{x}, \mathbf{z}, i)\right)\right| \\
& +\sum_{i: z_{i}=a, S_{i}=s, \ell(\mathbf{x}, \mathbf{z}, i) \in B} \frac{1}{n}\left|\log p_{x}\left(z_{i} \mid S_{i}\right)-\log \bar{p}_{\mathbf{x}}\left(z_{i}, \ell(\mathbf{x}, \mathbf{z}, i)\right)\right| \\
\leq & \frac{\epsilon N_{\mathbf{z}}(a, s)}{n}+\frac{C \log n}{n}\left|\left\{i: \ell(\mathbf{x}, \mathbf{z}, i) \in A_{n, a, s}(\delta)\right\}\right| \\
& +\frac{C \log n}{n}\left|\left\{i: z_{i}=a, S_{i}=s, \ell(\mathbf{x}, \mathbf{z}, i) \in B\right\}\right| .
\end{aligned}
$$

Therefore, we have

$$
\begin{array}{r}
\left|\bar{S}_{n}\left(q_{z}|| p_{x}\right)-\check{S}_{n}\left(q_{z}|| p_{x}\right)\right| \leq \epsilon+\frac{C \log n}{n}|\{i: \ell(\mathbf{x}, \mathbf{z}, i) \in B\}| \\
+\sum_{a \in \chi} \sum_{s \in \mathcal{S}} \frac{C \log n}{n}\left|\left\{i: \ell(\mathbf{x}, \mathbf{z}, i) \in A_{n, a, s}(\delta)\right\}\right| .
\end{array}
$$

In order to prove the convergence in probability, we only need to prove that, for any $\epsilon>0$,

$$
\operatorname{Pr}\left\{\frac{\log n}{n}\left|\left\{i: \ell(\mathbf{x}, \mathbf{z}, i) \in A_{n, a, s}(\delta)\right\}\right| \leq \epsilon\right\} \rightarrow 1
$$

and

$$
\operatorname{Pr}\left\{\frac{\log n}{n}|\{i: \ell(\mathbf{x}, \mathbf{z}, i) \in B\}| \leq \epsilon\right\} \rightarrow 1,
$$

as $n \rightarrow \infty$.

It follows from Lemma 3, choosing $\varepsilon=\epsilon \delta^{2}$,

$$
\begin{aligned}
\operatorname{Pr}\left\{\bigcup_{k}\left\{\frac{N_{\mathbf{z}}(k)}{n} \geq \frac{\varepsilon}{\log ^{3} n}\right\}\right\} & \leq \sum_{k} \operatorname{Pr}\left\{\frac{N_{\mathbf{z}}(k)}{n} \geq \frac{\varepsilon}{\log ^{3} n}\right\} \\
& \leq O\left(n^{-\frac{1}{2}} \log ^{3} n\right)
\end{aligned}
$$


Therefore

$$
\begin{aligned}
\operatorname{Pr}\left\{\max _{k} \frac{N_{\mathbf{z}}(k)}{n}<\frac{\varepsilon}{\log ^{3} n}\right\} & =\operatorname{Pr}\left\{\bigcap_{k}\left\{\frac{N_{\mathbf{z}}(k)}{n}<\frac{\varepsilon}{\log ^{3} n}\right\}\right\} \\
& \rightarrow 1,
\end{aligned}
$$

as $n \rightarrow \infty$. Then, (55) and (56) can be proved by combining (58) and (48), and the fact that $B$ is a finite set (i.e. $|B| \leq|\mathcal{S}|$ ).

Lemma 3 Under the same assumptions of Theorem 1, for any $\varepsilon>0$, and any $1 \leq k \leq n / w(n)$, where $w(n)=n^{\frac{1}{2}+\eta}\left(0<\eta<\frac{1}{2}\right)$, we have

$$
\operatorname{Pr}\left\{\frac{N_{\mathbf{z}}(k)}{n} \geq \frac{\varepsilon}{\log ^{3} n}\right\}=O\left(n^{\eta-1} \log ^{3} n\right) .
$$

Proof: In the BWT output, symbols from both $\mathbf{x}$ and $\mathbf{z}$ are sorted by context. In one segment, symbols from $\mathbf{x}$ and symbols from $\mathbf{z}$ have common context(s). Here, a context can be understood as a node in the context tree, which specifies a number of symbols occurring before the current symbol. The depth of the node equals the number of symbols the context specifies. The children of a node further specify more past symbols. The (infinite) sequence of past symbols is referred to as unbounded context. The uniform segmentation is determined by the realization $\mathbf{x}$, which partitions the leaves of the context tree into $n / w(n)$ classes, as shown in Figure 4. In this example, there are four segments. The first segment contains contexts "AA" and "AABA"; the second segment contains contexts "BABA" and "BBA"; the third segment contains contexts "AB" and "ABB"; the fourth segment contains context "BBB". The set of contexts of a segment is in fact represented by a set of nodes in the context tree. In order to determine the set of contexts of the $k$-th segment, we must find the boundary of the $k$-th segment and the $(k+1)$-th segment (as well as the boundary of the $(k-1)$-th segment and the $k$-th segment) in the context tree.

In the above, we have described an "ideal" non-overlapping partition of contexts based on uniform segmentation according to symbols from $\mathbf{x}$. However, if there are symbols (from $\mathbf{z}$ ) between the last symbol (from $\mathbf{x}$ ) of the $k$-th segment and the first symbol (from $\mathbf{x}$ ) of the $(k+1)$-th segment, we can not immediately determine which segment they should belong to according to the "ideal" partition. In our implementation of the algorithm, we resolve this by simply including those symbols from $\mathbf{z}$ into the previous segment. As our objective is to prove that the probability of $N_{\mathbf{z}}(k)$ being too large is very small, it suffices to prove it for an upper bound of $N_{\mathbf{z}}(k)$. So we modify the definition of the set of contexts of a segment, and allow a small overlapping across two consecutive segments. 


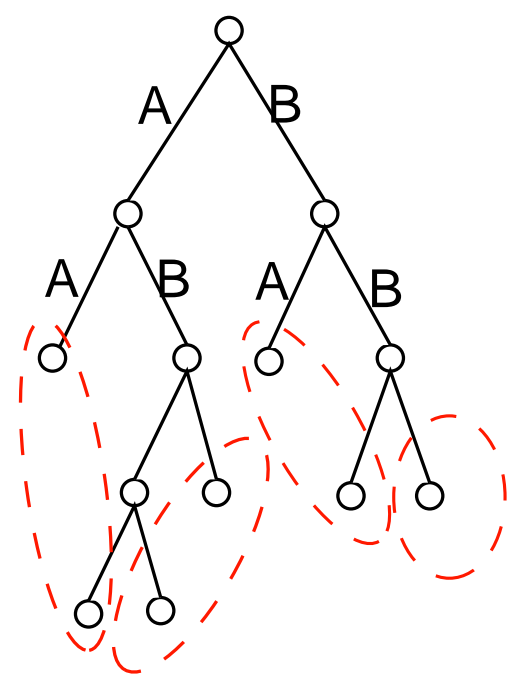

Fig. 4. "Ideal" partition of the context tree.

The set of contexts of the $k$-th segment can be determined by finding the first different symbol in the unbounded contexts of the first and second symbols (from $\mathbf{x}$ ) of the $(k+1)$-th segment and the first different symbol in the unbounded contexts of the last two symbols (from $\mathbf{x}$ ) of the $(k-1)$-th segment. Note that the set of contexts of the $k$-th segment under this definition is slightly larger than the set of contexts defined by the ideal partition.

Under the assumption that all conditional probabilities are less than 1 , there exists a constant $0<\xi<1$ such that $p_{x}(a \mid s) \leq \xi$ and $q_{z}(a \mid s) \leq \xi$ for any $a \in \chi$ and $s \in \mathcal{S}$.

Let us denote the stationary probability of contexts of the Markov source $p_{x}$ and the Markov source $q_{z}$ by $\mu_{x}$ and $\mu_{z}$ respectively. Let us consider the set of contexts $U_{n}(k)$, which is a set of nodes that are to the left of a boundary in the context tree, such that all the nodes included in $U_{n}(k)$ have depths no more than $l \triangleq\left\lceil\frac{\beta \log n}{\log \frac{1}{\xi}}\right\rceil$, where $0<\beta<\min \left\{\frac{\eta}{2}, \frac{1}{2}-\eta\right\}$ is a constant, and the following condition is satisfied: if $\frac{k w(n)}{n}+\frac{1}{n^{\beta}} \leq 1$

$$
\frac{k w(n)}{n}+\frac{1}{n^{\beta}} \leq \mu_{x}\left(U_{n}(k)\right) \leq \frac{k w(n)}{n}+\frac{2}{n^{\beta}}
$$

if $\frac{k w(n)}{n}+\frac{1}{n^{\beta}}>1$

$$
\mu_{x}\left(U_{n}(k)\right)=1
$$

Since the stationary distribution of a node of depth $\left\lceil\frac{\beta \log n}{\log \frac{1}{\xi}}\right\rceil$ is upper bounded by $\frac{1}{n^{\beta}}$, such $U_{n}(k)$ exists. Similarly, consider the set of contexts $L_{n}(k)$, which is a set of nodes that are to the left of a boundary in the context tree, such that all the nodes included in $L_{n}(k)$ have depths no more than $\left\lceil\frac{\beta \log n}{\log \frac{1}{\xi}}\right\rceil$, and the following condition is satisfied: 
if $\frac{(k-1) w(n)}{n}-\frac{1}{n^{\beta}} \geq 0$

$$
\frac{(k-1) w(n)}{n}-\frac{2}{n^{\beta}} \leq \mu_{x}\left(L_{n}(k)\right) \leq \frac{(k-1) w(n)}{n}-\frac{1}{n^{\beta}}
$$

if $\frac{(k-1) w(n)}{n}-\frac{1}{n^{\beta}}<0$

$$
\mu_{x}\left(L_{n}(k)\right)=0
$$

Let $N_{\mathbf{x}}\left(U_{n}(k)\right)$ denote the number of symbols in $\mathbf{x}$ that have context in $U_{n}(k)$ and $N_{\mathbf{x}}\left(L_{n}(k)\right)$ denote the number of symbols in $\mathbf{x}$ that have context in $L_{n}(k)$. Apparently, if $\mu_{x}\left(U_{n}(k)\right)=1$, then $N_{\mathbf{x}}\left(U_{n}(k)\right)=n$; if $\mu_{x}\left(L_{n}(k)\right)=0$, then $N_{\mathbf{x}}\left(L_{n}(k)\right)=0$. For the non-trivial cases, we have

$$
\mu_{x}\left(U_{n}(k)\right)=\frac{k w(n)}{n}+\frac{1}{n^{\delta_{1}}}
$$

and

$$
\mu_{x}\left(L_{n}(k)\right)=\frac{(k-1) w(n)}{n}-\frac{1}{n^{\delta_{2}}}
$$

where $\frac{1}{2} \beta<\delta_{1} \leq \beta$ and $\frac{1}{2} \beta<\delta_{2} \leq \beta$. Therefore, we have

$$
\begin{aligned}
& \operatorname{Pr}\left\{N_{\mathbf{x}}\left(U_{n}(k)\right) \leq k w(n)\right\} \\
& =\operatorname{Pr}\left\{N_{\mathbf{x}}\left(U_{n}(k)\right)-k w(n)-n^{1-\delta_{1}} \leq-n^{1-\delta_{1}}\right\} \\
& =\operatorname{Pr}\left\{N_{\mathbf{x}}\left(U_{n}(k)\right)-n \mu_{x}\left(U_{n}(k)\right) \leq-n^{1-\delta_{1}}\right\} \\
& \leq n^{2 \delta_{1}} E\left[\left(\frac{1}{n} N_{\mathbf{x}}\left(U_{n}(k)\right)-\mu_{x}\left(U_{n}(k)\right)\right)^{2}\right] \\
& =O\left(n^{2 \beta-1} l^{3}\right) \\
& =O\left(n^{2 \beta-1} \log ^{3} n\right)
\end{aligned}
$$

and

$$
\begin{aligned}
& \operatorname{Pr}\left\{N_{\mathbf{x}}\left(L_{n}(k)\right) \geq(k-1) w(n)\right\} \\
& =\operatorname{Pr}\left\{N_{\mathbf{x}}\left(L_{n}(k)\right)-(k-1) w(n)+n^{1-\delta_{2}} \geq n^{1-\delta_{2}}\right\} \\
& =\operatorname{Pr}\left\{N_{\mathbf{x}}\left(L_{n}(k)\right)-n \mu_{x}\left(L_{n}(k)\right) \geq n^{1-\delta_{2}}\right\} \\
& \leq n^{2 \delta_{2}} E\left[\left(\frac{1}{n} N_{\mathbf{x}}\left(L_{n}(k)\right)-\mu_{x}\left(L_{n}(k)\right)\right)^{2}\right] \\
& =O\left(n^{2 \beta-1} l^{3}\right) \\
& =O\left(n^{2 \beta-1} \log ^{3} n\right),
\end{aligned}
$$


where (69) and (74) follow from Lemma 5 in Appendix F; (70) and (75) follow from the fact that $l=\left\lceil\frac{\beta \log n}{\log \frac{1}{\xi}}\right\rceil$. Thus, we have

$$
\begin{aligned}
& \operatorname{Pr}\left\{\left\{N_{\mathbf{x}}\left(U_{n}(k)\right) \leq k w(n)\right\} \bigcup\left\{N_{\mathbf{x}}\left(L_{n}(k)\right) \geq(k-1) w(n)\right\}\right\} \\
& =O\left(n^{2 \beta-1} \log ^{3} n\right) .
\end{aligned}
$$

Let $M_{n}(k)$ be the context set that contains contexts in $U_{n}(k)$ but not in $L_{n}(k)$.

$$
M_{n}(k)=U_{n}(k) \backslash L_{n}(k) .
$$

From (60) and (62), we have

$$
\mu_{x}\left(M_{n}(k)\right) \leq \frac{1}{n^{\frac{1}{2}-\eta}}+\frac{4}{n^{\beta}} \leq \frac{5}{n^{\beta}}
$$

where $0<\beta<\min \left\{\frac{\eta}{2}, \frac{1}{2}-\eta\right\}$.

Next, we establish the connection between $\mu_{x}$ and $\mu_{z}$. Let $T_{n}(k)$ be any set of contexts. As shown in Figure 5, we find the last symbol in common of these contexts, which corresponds to the latest common ancestor of the set of nodes specified by $T_{n}(k)$. Then, the set $T_{n}(k)$ can be divided into two disjoint sets $T_{n}(k)=T_{n}^{L}(k) \cup T_{n}^{R}(k)$, which are in different branches of the common ancestor (the division is not unique if more than two branches of the common ancestor are involved). Denote the common ancestor of the nodes in $T_{n}^{L}(k)$ by $P^{L}(k)$, the node $P^{L}(k)$ 's rightmost child in $T_{n}^{L}(k)$ by $C^{L}(k)$. Let $\Lambda\left(P^{L}(k)\right)$ be the set of nodes including the node $P^{L}(k)$ and all its offsprings. Then we have $C^{L}(k) \in T_{n}^{L}(k) \subseteq \Lambda\left(P^{L}(k)\right)$. Let

$$
\tau=\min _{a \in \chi s \in \mathcal{S}}\left\{p_{x}(a \mid s): p_{x}(a \mid s)>0\right\}
$$

and

$$
\max _{a \in \chi s \in \mathcal{S}}\left\{q_{z}(a \mid s)\right\} \leq \xi<1 .
$$

If $\mu_{x}\left(C^{L}(k)\right)=0$, we take the node $C^{L}(k)$ out of the set $T_{n}^{L}(k)$ and find the new common ancestor of nodes in $T_{n}^{L}(k)$. If $\mu_{x}\left(T_{n}^{L}(k)\right)=0$, we exclude all the nodes in $T_{n}^{L}(k)$ from the set $T_{n}(k)$ and find the new common ancestor of nodes in $T_{n}(k)$. We repeat this until $\mu_{x}\left(C^{L}(k)\right)>0$ is satisfied. Now suppose $P^{L}(k)$ is of depth $l$, we have $\mu_{x}\left(T_{n}^{L}(k)\right) \geq C_{1} \tau^{l+1}$ and $\mu_{z}\left(T_{n}^{L}(k)\right) \leq C_{2} \xi^{l}$, where $C_{1}>0$ and $C_{2}>0$ are constants. Therefore, we have

$$
\mu_{z}\left(T_{n}^{L}(k)\right) \leq C_{3} \mu_{x}\left(T_{n}^{L}(k)\right)^{\gamma}
$$

where $\gamma=\frac{\log \xi}{\log \tau}$ (Note $0<\gamma<1$ ). Similarly, denote the common ancestor of the nodes in $T_{n}^{R}(k)$ by $P^{R}(k)$, its leftmost child in $P^{R}(k)$ by $C^{R}(k)$. Let $\Lambda\left(P^{R}(k)\right)$ be the set of nodes including the node $P^{R}(k)$ 
and all its offsprings. Then we have $C^{R}(k) \in T_{n}^{R}(k) \subseteq \Lambda\left(P^{R}(k)\right)$ and $\mu_{x}\left(C^{R}(k)\right)>0$. Thus

$$
\mu_{z}\left(T_{n}^{R}(k)\right) \leq C_{4} \mu_{x}\left(T_{n}^{R}(k)\right)^{\gamma}
$$

Combining (81) and (82), we conclude that for any context set $T_{n}(k)$,

$$
\mu_{z}\left(T_{n}(k)\right) \leq C_{5} \mu_{x}\left(T_{n}(k)\right)^{\gamma}
$$

where $C_{5}=C_{3}+C_{4}$. Combining (78) and (83), we have

$$
\mu_{z}\left(M_{n}(k)\right) \leq \frac{C_{5} 5^{\gamma}}{n^{\beta \gamma}}
$$

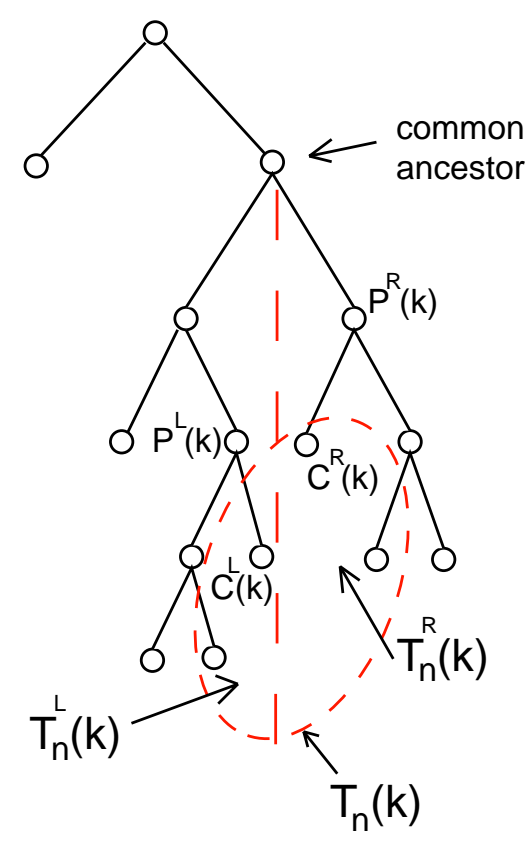

Fig. 5. The set of contexts $T_{n}(k)$ is divided into two disjoint sets $T_{n}^{L}(k)$ and $T_{n}^{R}(k)$. 
Finally, we have

$$
\begin{array}{rl}
\operatorname{Pr}\left\{\frac{N_{\mathbf{z}}(k)}{n} \geq \frac{\varepsilon}{\log ^{3} n}\right\} \\
\leq & \operatorname{Pr}\left\{\left\{N_{\mathbf{x}}\left(U_{n}(k)\right) \leq k w(n)\right\} \bigcup\left\{N_{\mathbf{x}}\left(L_{n}(k)\right) \geq(k-1) w(n)\right\}\right\} \\
& +\operatorname{Pr}\left\{\left\{N_{\mathbf{x}}\left(U_{n}(k)\right)>k w(n)\right\} \bigcap\left\{N_{\mathbf{x}}\left(L_{n}(k)\right)<(k-1) w(n)\right\}\right. \\
& \left.\bigcap\left\{\frac{N_{\mathbf{z}}(k)}{n} \geq \frac{\varepsilon}{\log ^{3} n}\right\}\right\} \\
\leq & \operatorname{Pr}\left\{\left\{N_{\mathbf{x}}\left(U_{n}(k)\right)>k w(n)\right\} \bigcap\left\{N_{\mathbf{x}}\left(L_{n}(k)\right)<(k-1) w(n)\right\}\right. \\
& \left.\bigcap\left\{\frac{N_{\mathbf{z}}\left(M_{n}(k)\right)}{n} \geq \frac{\varepsilon}{\log ^{3} n}\right\}\right\}+O\left(n^{2 \beta-1} \log ^{3} n\right) \\
\leq & \operatorname{Pr}\left\{\frac{N_{\mathbf{z}}\left(M_{n}(k)\right)}{n} \geq \frac{\varepsilon}{\log ^{3} n}\right\}+O\left(n^{2 \beta-1} \log ^{6} n\right) \\
\leq & \operatorname{Pr}\left\{\frac{N_{\mathbf{z}}\left(M_{n}(k)\right)}{n}-\mu_{z}\left(M_{n}(k)\right) \geq \frac{\varepsilon}{\log ^{3} n}-\frac{C_{5} 5^{\gamma}}{n^{\beta \gamma}}\right\}+O\left(n^{2 \beta-1} \log ^{3} n\right) \\
\leq & E\left[\left(\frac{1}{n} N_{\mathbf{z}}\left(M_{n}(k)\right)-\mu_{z}\left(M_{n}(k)\right)\right)^{2}\right] \\
\left(\frac{\varepsilon}{\log ^{3} n}-\frac{C_{5} 5^{\beta \gamma}}{n^{\beta \gamma}}\right)^{2} & O\left(n^{2 \beta-1} \log ^{3} n\right) \\
= & O\left(n^{-1} \log ^{9} n\right)+O\left(n^{2 \beta-1} \log ^{3} n\right) \\
= & O\left(n^{\eta-1} \log ^{3} n\right)
\end{array}
$$

where (86) follows from (76) and the fact that $N_{\mathbf{z}}\left(M_{n}(k)\right) \geq N_{\mathbf{z}}(k)$ holds when both $N_{\mathbf{x}}\left(U_{n}(k)\right)>k w(n)$ and $N_{\mathbf{x}}\left(L_{n}(k)\right)<(k-1) w(n)$ are satisfied; (88) follows from (84); (90) follows from Lemma 5 in Appendix F; and (91) follows from the fact that $0<\beta<\min \left\{\frac{\eta}{2}, \frac{1}{2}-\eta\right\}$.

\section{Divergence ESTIMATOR Via CONTEXT MODELING}

\section{A. Overview}

Universal arithmetic-coding based data compression algorithms, such as PPM [6] and CTW [13], [14], use adaptive statistical modeling methods. These methods update a tree-based context model, which assigns a probability to the next symbol given the past.

While it is obvious that the probability estimates fed to the arithmetic coder can be used to estimate entropy, we show in this section that the above probability estimation and context model updating mechanisms can also be modified to provide an alternative way to estimate divergence. The structure of the modified algorithm is shown in Figure 6. 


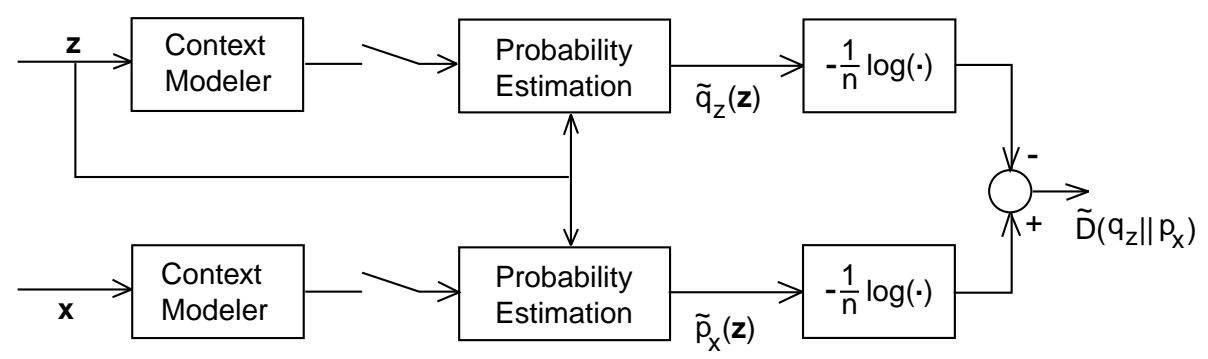

Fig. 6. Divergence estimator via context modeling.

We first build and update context models for both sequences $\mathbf{z}$ and $\mathbf{x}$. Once the whole sequences $\mathbf{z}$ and $\mathrm{x}$ have been observed and their modeling has been completed, the switches in Figure 6 are closed, the estimated models are fixed and we estimate both $\tilde{q}_{\mathbf{z}}\left(z^{n}\right)$ and $\tilde{p}_{\mathbf{x}}\left(z^{n}\right)$. We then take the difference of the normalized logarithm of $\tilde{q}_{\mathbf{z}}\left(z^{n}\right)$ and $\tilde{p}_{\mathbf{x}}\left(z^{n}\right)$ as the divergence estimate $\tilde{D}\left(q_{z} \| p_{x}\right)$. The universal context modeler can take many different embodiments leading to different algorithms. In this paper we focus attention on the Context Tree Weighting (CTW) method. Note that an alternative to the divergence estimator in Figure 6 which leads to slightly degraded estimation consists of generating $\tilde{q}_{\mathbf{z}}\left(z^{n}\right)$ "on the fly" at the same time as the context is updated, as is done in arithmetic-coding based compression where the probability estimate must be computed causally.

\section{B. The Context Tree Weighting method}

The CTW method uses a weighting scheme to provide a weighted probability [13], which is a mixture of estimated probabilities for different models. When the context modeler uses CTW, the lower branch in Figure 6 is quite similar to the model-freezing method used in [7]. Note that in [7], the idea of freezing the model is important in estimating $\tilde{p}_{\mathbf{x}}\left(z^{n}\right)$, since sequence $\mathbf{z}$ should not affect the statistical model learned from sequence $\mathbf{x}$. Experimental results on texts classification using CTW with and without model-freezing have been shown in [7].

In this description, for simplicity, the sources are assumed to be binary. The basic CTW method [13] assumes that the maximum memory length $D$ is known and also past symbols $x_{1-D}^{0}$ are known in addition to $x_{1}^{n}$. An example of a context tree with $D=3$ is shown in Fig. 7. Each node in the tree corresponds to a context. Counts $a_{s}$ and $b_{s}$ stored in node $s$ are the number of 0's and 1's emitted from the corresponding context. For node $s$, the estimated probability $P_{e}^{s}=P_{e}\left(a_{s}, b_{s}\right)$ is the Krichevsky-Trofimov probability estimate of a sequence containing $a_{s}$ zeros and $b_{s}$ ones, which is defined as follows: $P_{e}(0,0)=1$ and for 
$a \geq 0$ and $b \geq 0$

$$
P_{e}(a+1, b)=\frac{a+\frac{1}{2}}{a+b+1} \cdot P_{e}(a, b)
$$

and

$$
P_{e}(a, b+1)=\frac{b+\frac{1}{2}}{a+b+1} \cdot P_{e}(a, b)
$$

In node $s$, the conditional estimated probabilities for zero and one are $\frac{a_{s}+\frac{1}{2}}{a_{s}+b_{s}+1}$ and $\frac{b_{s}+\frac{1}{2}}{a_{s}+b_{s}+1}$ respectively. The weighted probability $P_{w}^{s}$ of node $s$ is calculated as follows:

$$
P_{w}^{s} \triangleq\left\{\begin{aligned}
\frac{1}{2} P_{e}^{s}+\frac{1}{2} P_{w}^{0 s} P_{w}^{1 s} & : \quad 0 \leq l(s) \leq D, \\
P_{e}^{s} & : l(s)=D,
\end{aligned}\right.
$$

where the nodes $0 s$ and $1 s$ are the children of node $s$, and $l(s)$ is the depth of node $s$. When we build the context tree from sequence $x_{1}^{n}$, we add one symbol at a time. In adding symbol $x_{t}$, we have to update the counts $a_{s}$ and $b_{s}$, the estimated probability $P_{e}^{s}$ and the weighted probability $P_{w}^{s}$ for each context $s$ of $x_{t}$. The order of updating is from the context of the longest depth (a leaf node) to the root. The limitation is that with fixed maximum memory length $D$ we can only learn statistical models of order no more than $D$.

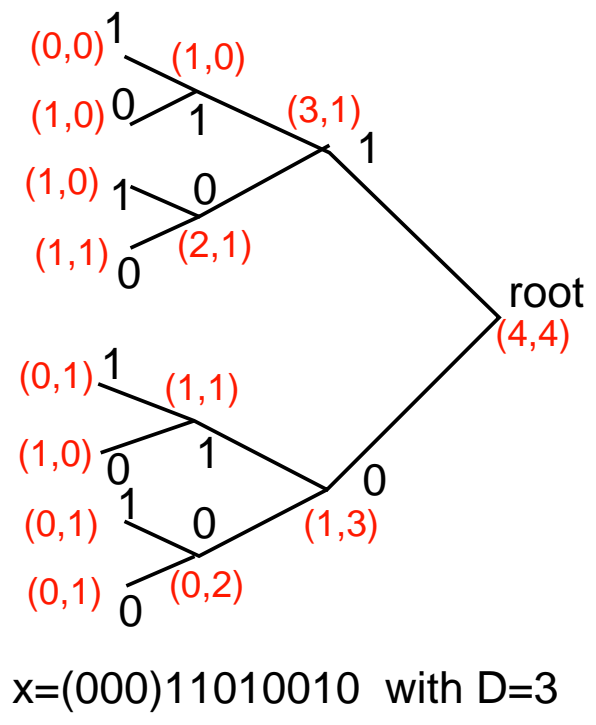

Fig. 7. The basic CTW method with $D=3$ and $x_{-2}^{8}=00011010010$. Counts $\left(a_{s}, b_{s}\right)$ are stored in each node $s$.

The extended CTW method [14] assumes unbounded memory length, and therefore the depth of the context tree is unbounded and grows with the length $n$. The sources are assumed to be binary. We preappend $\cdots \varepsilon \varepsilon \varepsilon$ before $x_{1}^{n}$ as unknown symbols. The extended CTW method stores all relevant statistical information of different orders provided by the sequence $x_{1}^{n}$. When a certain context has occurred only 
once in the sequence $x_{1}^{n}$ (i.e., there is only one symbol emitted from that context), the counts of the corresponding node sum up to 1 , in which case it is meaningless to further store counts of its children. Eventually, we always encounter a context that has never occurred before, because the context $\varepsilon x_{1}^{n}$ has never occurred before.

Definition: Let $s$ be a node of the context tree of $x_{1}^{n}$. Node $s$ is said to be a unique node of the context tree of $x_{1}^{n}$, if the corresponding subsequence occurs only once in $\varepsilon x_{1} x_{2} \cdots x_{n}$. Node $s$ is said to be a null node, if the corresponding subsequence has not occurred in $\varepsilon x_{1} x_{2} \cdots x_{n}$.

As we have discussed, it is unnecessary to maintain further children nodes of a unique node. But as we proceed on, a unique node at time $t$ might branch out at a later time. So we have to keep the position of the occurrence of the unique subsequence within $x_{1}^{t}$. In building the context tree, when we add the next symbol $x_{t}$ that has the current context $x_{1}^{t-1}$ to the context tree, we have to travel from the root, along nodes $x_{t-1}, x_{t-2}^{t-1}, x_{t-3}^{t-1}, \ldots$ until we encounter a null node (which means this node has never occurred before). The path from this null node to the root is called the updating path of the context $x_{1}^{t-1}$. After the addition, this null node becomes a unique node (that has occurred once). Then we should travel back to the root, updating the counts, the estimated probability and the weighted probability in every node in the updating path. The context tree and the updating process are shown in Fig. 8. The counts of 0's and 1's are shown in Fig. 9. The weighted probability is defined as follows.
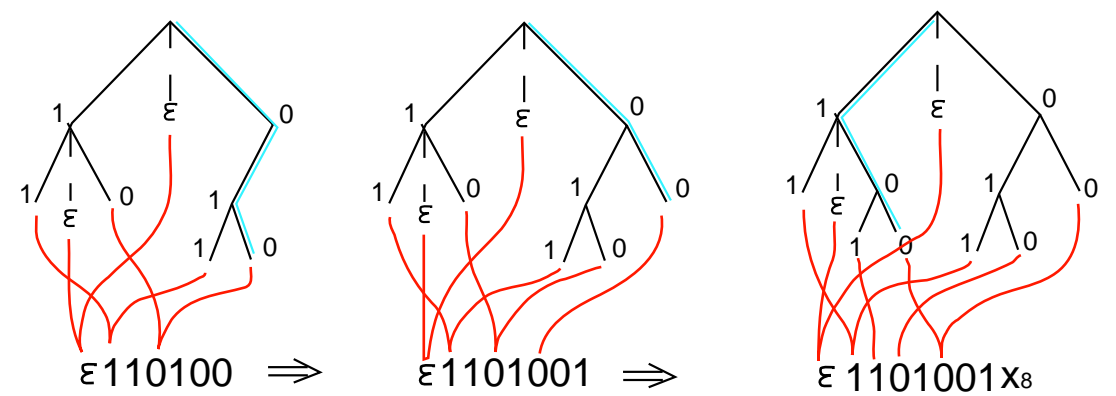

Fig. 8. Context tree updating with $x_{1}^{6}=110100$ and $x_{7}=1$. Red lines point from unique nodes to the occurrence in the sequence. Null nodes are not drawn. Blue lines are the last updating paths.

$$
P_{w}^{s}=\left\{\begin{aligned}
\frac{1}{2} P_{e}^{s}+\frac{1}{2} P_{w}^{0 s} P_{w}^{1 s} P_{w}^{\varepsilon s} & \text { if } s \text { is not a unique node } \\
\frac{1}{2} & \text { if } s \text { is a unique node }
\end{aligned}\right.
$$

When processing $x_{t}$, only those nodes in the updating path of $x_{1}^{t-1}$ need to be updated. For unique node $u, P_{w}^{u}=\frac{1}{2}$. For any internal node $s$ in the updating path (including the root), we have to re-calculate $P_{w}^{s}$ recursively. Notice, $P_{w}^{\varepsilon s}=1$ if $s$ is not a prefix of $x_{1}^{n}$ ( $\varepsilon s$ is a null node); and $P_{w}^{\varepsilon s}=\frac{1}{2}$ if $s$ is a prefix of $x_{1}^{n}$. 


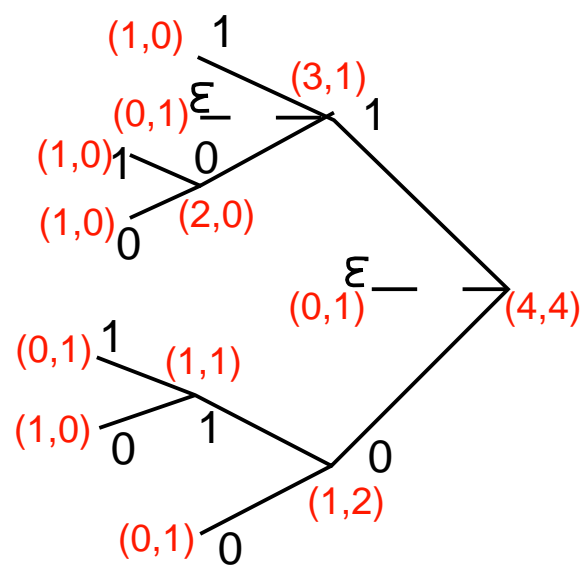

$X=\varepsilon 11010010$

Fig. 9. The extended CTW method with $\varepsilon x_{1}^{8}=\varepsilon 11010010$. Counts $\left(a_{s}, b_{s}\right)$ are stored in each node $s$.

Define the quantity $\beta^{s}\left(x_{1}^{n}\right)$ for node $s$ as follows. For the basic CTW method,

$$
\beta^{s}\left(x_{1}^{n}\right) \triangleq \frac{P_{e}^{s}\left(x_{1}^{n}\right)}{P_{w}^{0 s}\left(x_{1}^{n}\right) P_{w}^{1 s}\left(x_{1}^{n}\right)},
$$

for the extended CTW method,

$$
\beta^{s}\left(x_{1}^{n}\right) \triangleq \frac{P_{e}^{s}\left(x_{1}^{n}\right)}{P_{w}^{0 s}\left(x_{1}^{n}\right) P_{w}^{1 s}\left(x_{1}^{n}\right) P_{w}^{\varepsilon s}\left(x_{1}^{n}\right)} .
$$

C. Algorithms

\section{Algorithm with Basic CTW:}

1) Build the context tree based on the sequence $x_{1}^{n}$ using the basic CTW method.

2) Once the modeling is completed, do not allow any changes of the counts, the estimated probability $P_{e}^{s}$ or the weighted probability $P_{w}^{s}$ in any node $s$.

3) For each $i=1,2, \ldots, n$, estimate $\tilde{p}_{\mathbf{x}}\left(z_{i} \mid z_{i-D}^{i-1}\right)$ based on the above context tree independently, as follows:

a) Find the node $v$ of depth $D$ corresponding to context $z_{i-D}^{i-1}$.

b) Initialize $P_{w}^{v}\left(z_{i} \mid \cdot\right)=P_{e}^{v}\left(z_{i} \mid \cdot\right)$.

c) Travel from $v$ back to the root. For each node $s$ in the updating path,

$$
P_{w}^{s}\left(z_{i} \mid \cdot\right)=\left\{\begin{array}{r}
\frac{\beta^{s}\left(x_{1}^{n}\right)}{\beta^{s}\left(x_{1}^{n}\right)+1} P_{e}^{s}\left(z_{i} \mid \cdot\right)+\frac{1}{\beta^{s}\left(x_{1}^{n}\right)+1} P_{w}^{1 s}\left(z_{i} \mid \cdot\right) \\
\text { if } 1 s \text { is in the updating path } \\
\frac{\beta^{s}\left(x_{1}^{n}\right)}{\beta^{s}\left(x_{1}^{n}\right)+1} P_{e}^{s}\left(z_{i} \mid \cdot\right)+\frac{1}{\beta^{s}\left(x_{1}^{n}\right)+1} P_{w}^{0 s}\left(z_{i} \mid \cdot\right) \\
\text { if } 0 s \text { is in the updating path. }
\end{array}\right.
$$


d) $\tilde{p}_{\mathbf{x}}\left(z_{i} \mid z_{i-D}^{i-1}\right)=P_{w}^{\lambda}\left(z_{i} \mid \cdot\right)$.

4) Add up the estimates and subtract the entropy estimate (cf. Figure 6):

$$
\begin{aligned}
\tilde{D}\left(q_{z} \| p_{x}\right) & =-\frac{1}{n} \log _{2} \tilde{p}_{\mathbf{x}}\left(z_{1}^{n}\right)+\frac{1}{n} \log _{2} \tilde{q}_{\mathbf{z}}\left(z_{1}^{n}\right) \\
& =-\frac{1}{n} \sum_{i=1}^{n} \log _{2}\left(\tilde{p}_{\mathbf{x}}\left(z_{i} \mid z_{i-D}^{i-1}\right)\right)+\frac{1}{n} \log _{2} \tilde{q}_{\mathbf{z}}\left(z_{1}^{n}\right) .
\end{aligned}
$$

\section{Algorithm with Extended CTW:}

1) Build the context tree based on the sequence $x_{1}^{n}$ using the extended CTW method.

2) Once the modeling is completed, do not allow any changes of the counts or $P_{e}^{s}$ and $P_{w}^{s}$ in any node $s$.

3) For each $i=1,2, \ldots, n$, estimate $\tilde{p}_{\mathbf{x}}\left(z_{i} \mid z_{1}^{i-1}\right)$ based on the above context tree independently, as follows:

a) Find the longest suffix of $z_{1}^{i-1}$ which occurred as a context in $x_{1}^{n}$, which is equivalent to find the non-null node of the longest depth corresponding to this context. Let this node be $v^{\prime}$.

b) Initialize $P_{w}^{v^{\prime}}\left(z_{i} \mid \cdot\right)=P_{e}^{v^{\prime}}\left(z_{i} \mid \cdot\right)$.

c) Travel from $v^{\prime}$ back to the root. For each node $s$ in the updating path,

$$
P_{w}^{s}\left(z_{i} \mid \cdot\right)=\left\{\begin{array}{r}
\frac{\beta^{s}\left(x_{1}^{n}\right)}{\beta^{s}\left(x_{1}^{n}\right)+1} P_{e}^{s}\left(z_{i} \mid \cdot\right)+\frac{P_{w}^{1 s}\left(z_{i} \mid \cdot\right)}{\beta^{s}\left(x_{1}^{n}\right)+1} \\
\text { if } 1 s \text { is in the updating path, } \\
\frac{\beta^{s}\left(x_{1}^{n}\right)}{\beta^{s}\left(x_{1}^{n}\right)+1} P_{e}^{s}\left(z_{i} \mid \cdot\right)+\frac{P_{w}^{0 s}\left(z_{i} \mid \cdot\right)}{\beta^{s}\left(x_{1}^{n}\right)+1} \\
\text { if } 0 s \text { is in the updating path. }
\end{array}\right.
$$

d) $\tilde{p}_{\mathbf{x}}\left(z_{i} \mid z_{1}^{i-1}\right)=P_{w}^{\lambda}\left(z_{i} \mid \cdot\right)$.

4) Add up the estimates and subtract the entropy estimate.

$$
\begin{aligned}
\tilde{D}\left(q_{z}|| p_{x}\right) & =-\frac{1}{n} \log _{2} \tilde{p}_{\mathbf{x}}\left(z_{1}^{n}\right)+\frac{1}{n} \log _{2} \tilde{q}_{\mathbf{z}}\left(z_{1}^{n}\right) \\
& =-\frac{1}{n} \sum_{i=1}^{n} \log _{2}\left(\tilde{p}_{\mathbf{x}}\left(z_{i} \mid z_{1}^{i-1}\right)\right)+\frac{1}{n} \log _{2} \tilde{q}_{\mathbf{z}}\left(z_{1}^{n}\right) .
\end{aligned}
$$

It is suggested in [14] that a string of non-unique nodes without branches except the farthest one from the root are equivalent and can be replaced by a single super-node. Equivalent nodes all have the same counts and therefore the same estimated probability. Only the weighted probability corresponding to the node closest to the root is actually needed by its parent and therefore stored in the super-node. Suppose the 
number of nodes in the super-node $s$ is $d(s)$ and $P_{w}^{s}$ is the weighted probability stored in the super-node $s$. We have

$$
\begin{gathered}
P_{w}^{s}\left(x_{1}^{n}\right)=\left(1-\frac{1}{2^{d(s)}}\right) P_{e}^{s}\left(x_{1}^{n}\right)+\frac{1}{2^{d(s)}} P_{w}^{0 s}\left(x_{1}^{n}\right) P_{w}^{1 s}\left(x_{1}^{n}\right) P_{w}^{\varepsilon s}\left(x_{1}^{n}\right) \\
\beta^{s}\left(x_{1}^{n}\right)=\frac{\left(2^{d(s)}-1\right) P_{e}^{s}\left(x_{1}^{n}\right)}{P_{w}^{0 s}\left(x_{1}^{n}\right) P_{w}^{1 s}\left(x_{1}^{n}\right) P_{w}^{\varepsilon s}\left(x_{1}^{n}\right)}
\end{gathered}
$$

and

$$
\frac{\beta^{s}\left(x_{1}^{n}\right)}{\beta^{s}\left(x_{1}^{n}\right)+1}=\frac{\left(1-2^{-d(s)}\right) P_{e}^{s}\left(x_{1}^{n}\right)}{P_{w}^{s}\left(x_{1}^{n}\right)} .
$$

Then the updating rule (100) in step 3(c) holds for super-nodes. The compact context tree implementation apparently has the advantage of using fewer nodes and fewer operations.

\section{Analysis}

It is easy to prove the convergence of the entropy estimator via CTW. The cross term estimator is more problematic and so we focus on the estimation of the cross term via the basic CTW method.

After building the context tree based on the sequence $x_{1}^{n}$, we proceed to estimate $-\frac{1}{n} \log _{2} \tilde{p}_{\mathbf{x}}\left(z_{1}^{n}\right)$ by estimating $n$ terms $\tilde{p}_{\mathbf{x}}\left(z_{i} \mid z_{i-D}^{i-1}\right)=P_{w}^{\lambda}\left(z_{i} \mid \cdot\right), i=1,2, \ldots, n$. In order to bound the aggregate error

$$
\left|-\frac{1}{n} \log _{2} \tilde{p}_{\mathbf{x}}\left(z_{1}^{n}\right)+\frac{1}{n} \log _{2} p_{x}\left(z_{1}^{n}\right)\right|,
$$

we bound the error

$$
\left|\log _{2} \tilde{p}_{\mathbf{x}}\left(z_{i} \mid z_{i-D}^{i-1}\right)-\log _{2} p_{x}\left(z_{i} \mid z_{i-D}^{i-1}\right)\right|
$$

for any $z_{i} \in \chi$ and $z_{i-D}^{i-1} \in \chi^{D}$.

The estimate $\tilde{p}_{\mathbf{x}}\left(z_{i} \mid z_{i-D}^{i-1}\right)$ is obtained by using the same context tree updating rule, where $z_{i}$ is the next symbol and $z_{i-D}^{i-1}$ is the current context. If we were not freezing the context tree, $P_{w}^{\lambda}\left(x_{1}^{n}\right)$ would be updated to include the new incoming symbol $z_{i}$ and would become $P_{w}^{\lambda}\left(x_{1}^{n}, z_{i}\right)$. The conditional weighted probability at the root $P_{w}^{\lambda}\left(z_{i} \mid z_{i-D}^{i-1}\right)=P_{w}^{\lambda}\left(x_{1}^{n}, z_{i}\right) / P_{w}^{\lambda}\left(x_{1}^{n}\right)$ is the probability estimate we need. We emphasize that once the context tree modeling is completed, we do not change any counts $\left(a_{s}, b_{s}\right)$ or $P_{e}^{s}$ and $P_{w}^{s}$ stored in the context tree; i.e, we just need to obtain $P_{w}^{\lambda}\left(x_{1}^{n}, z_{i}\right)$ by computing $P_{w}^{s}\left(x_{1}^{n}, z_{i}\right)$ along the updating path of $z_{i-D}^{i-1}$.

Our main consistency result for the algorithm in Section IV-C is the following: 
Theorem 2 Let $\mathbf{z}$ and $\mathbf{x}$ be sequences of length $n$ generated from finite-alphabet finite-state d-th order Markov sources $q_{z}$ and $p_{x}$ respectively. Let $\tilde{D}_{n}\left(q_{z} \| p_{x}\right)$ denote our divergence estimator based on the basic CTW method of maximum memory length $D(99)$, where $D \geq d$. Then

$$
\lim _{n \rightarrow \infty} \tilde{D}_{n}\left(q_{z}|| p_{x}\right)=D\left(q_{z}|| p_{x}\right) \quad \text { a.s. }
$$

Proof: We prove the convergence of the estimate of the cross term. The same argument applies to the entropy term.

Let us examine the updating computation for $z_{i}$, where $1 \leq i \leq n$. For an internal node $s$ in the updating path, if $1 s$ is in the updating path,

$$
\begin{aligned}
& P_{w}^{s}\left(z_{i} \mid z_{i-D}^{i-1}\right) \\
& =\frac{P_{w}^{s}\left(x_{1}^{n}, z_{i}\right)}{P_{w}^{s}\left(x_{1}^{n}\right)} \\
& =\frac{\frac{1}{2} P_{e}^{s}\left(x_{1}^{n}\right) P_{e}^{s}\left(z_{i} \mid z_{i-D}^{i-1}\right)+\frac{1}{2} P_{w}^{0 s}\left(x_{1}^{n}\right) P_{w}^{1 s}\left(x_{1}^{n}\right) P_{w}^{1 s}\left(z_{i} \mid z_{i-D}^{i-1}\right)}{P_{w}^{s}\left(x_{1}^{n}\right)} \\
& =\frac{\beta^{s}\left(x_{1}^{n}\right)}{\beta^{s}\left(x_{1}^{n}\right)+1} P_{e}^{s}\left(z_{i} \mid z_{i-D}^{i-1}\right)+\frac{1}{\beta^{s}\left(x_{1}^{n}\right)+1} P_{w}^{1 s}\left(z_{i} \mid z_{i-D}^{i-1}\right),
\end{aligned}
$$

if $0 s$ is in the updating path,

$$
\begin{aligned}
& P_{w}^{s}\left(z_{i} \mid z_{i-D}^{i-1}\right) \\
& =\frac{\frac{1}{2} P_{e}^{s}\left(x_{1}^{n}\right) P_{e}^{s}\left(z_{i} \mid z_{i-D}^{i-1}\right)+\frac{1}{2} P_{w}^{0 s}\left(x_{1}^{n}\right) P_{w}^{1 s}\left(x_{1}^{n}\right) P_{w}^{0 s}\left(z_{i} \mid z_{i-D}^{i-1}\right)}{P_{w}^{s}\left(x_{1}^{n}\right)} \\
& =\frac{\beta^{s}\left(x_{1}^{n}\right)}{\beta^{s}\left(x_{1}^{n}\right)+1} P_{e}^{s}\left(z_{i} \mid z_{i-D}^{i-1}\right)+\frac{1}{\beta^{s}\left(x_{1}^{n}\right)+1} P_{w}^{0 s}\left(z_{i} \mid z_{i-D}^{i-1}\right) .
\end{aligned}
$$

For a leaf node $v$ in the updating path,

$$
P_{w}^{v}\left(z_{i} \mid z_{i-D}^{i-1}\right)=P_{e}^{v}\left(z_{i} \mid z_{i-D}^{i-1}\right)
$$

This computation starts with a leaf and is repeated recursively along the updating path, until we reach the root and obtain $P_{w}^{\lambda}\left(z_{i} \mid z_{i-D}^{i-1}\right)$. So $P_{w}^{\lambda}\left(z_{i} \mid z_{i-D}^{i-1}\right)$ is a weighted sum of $P_{e}^{s}\left(z_{i} \mid z_{i-D}^{i-1}\right)$, where $s$ is any node in the updating path. Let $\{s \rightarrow \lambda\}$ denote the set of nodes in the path from $s$ to the root. The weight associated with $P_{e}^{s}\left(z_{i} \mid z_{z-D}^{z-1}\right)$ is $\beta^{s}\left(x_{1}^{n}\right) \prod_{u \in\{s \rightarrow \lambda\}} \frac{1}{\beta^{u}\left(x_{1}^{n}\right)+1}$, where $s$ is an internal node in the updating path. The weight associated with $P_{w}^{v}\left(z_{i} \mid z_{i-D}^{i-1}\right)$, where $v$ is the leaf in the updating path, is $\prod_{u \in\{\{v \rightarrow \lambda\} \backslash v\}} \frac{1}{\beta^{u}\left(x_{1}^{n}\right)+1}$. Suppose $z_{i-t_{i}}^{i-1}$ is the suffix of $z_{i-D}^{i-1}$ that is a state of the finite memory source, where $t_{i}$ is less than $D$ (the upper bound on the memory length). Suppose $s^{0}, s^{1}, \ldots, s^{t_{i}-1}$ are the $t_{i}$ nodes in the updating path with depth less than $t_{i}$ (including the root $\lambda$ ). Since $s^{0}, \ldots, s^{t_{i}-1}$ have depth less than $t_{i}, P_{e}^{s^{0}}\left(z_{i} \mid \cdot\right), \ldots, P_{e}^{s^{t_{i}-1}}\left(z_{i} \mid \cdot\right)$ do not converge to $p_{x}\left(z_{i} \mid z_{i-D}^{i-1}\right)$ and should not be included in the weighted 
conditional probability. Lemma 4 below implies that the contribution of $P_{e}^{s^{0}}\left(z_{i} \mid \cdot\right), \ldots, P_{e}^{s^{t_{i}-1}}\left(z_{i} \mid \cdot\right)$ to the weighted conditional probability $P_{w}^{\lambda}\left(z_{i} \mid z_{i-D}^{i-1}\right)$ converges to zero. On the other hand, $P_{e}^{s^{t_{i}}}\left(z_{i} \mid \cdot\right), \ldots, P_{e}^{s^{D}}\left(z_{i} \mid \cdot\right)$ all converge to $p_{x}\left(z_{i} \mid z_{i-D}^{i-1}\right)$ almost surely, therefore the weighted conditional probability $P_{w}^{\lambda}\left(z_{i} \mid \cdot\right)$ converges to $p_{x}\left(z_{i} \mid z_{i-D}^{i-1}\right)$ almost surely as $n \rightarrow \infty$. Thus, we have

$$
\left|-\frac{1}{n} \log _{2} \tilde{p}_{\mathbf{x}}\left(z_{1}^{n}\right)+\frac{1}{n} \log _{2} p_{x}\left(z_{1}^{n}\right)\right| \rightarrow 0
$$

almost surely. Combined with the fact that $-\frac{1}{n} \log _{2} p_{x}\left(z_{1}^{n}\right) \rightarrow S\left(q_{z} \| p_{x}\right)$ almost surely, we have

$$
\lim _{n \rightarrow \infty}-\frac{1}{n} \log _{2} \tilde{p}_{\mathbf{x}}\left(z_{1}^{n}\right)=S\left(q_{z} \| p_{x}\right) \quad \text { a.s. }
$$

Applying the same argument to the estimate of the entropy term, we obtain (105).

Lemma 4 Suppose $s$ is an internal node in the tree representation of the source, but s itself is not a state of the source (i.e. offsprings of the node s do not all have the same conditional probabilities). Then

$$
\lim _{n \rightarrow \infty} \beta^{s}\left(x_{1}^{n}\right)=0 \quad \text { a.s. }
$$

Proof:

$$
\begin{aligned}
& \frac{\beta^{s}\left(x_{1}^{n}\right)}{\beta^{s}\left(x_{1}^{n}\right)+1} \\
& =\frac{P_{e}^{s}\left(x_{1}^{n}\right)}{2 P_{w}^{s}\left(x_{1}^{n}\right)} \\
& \leq \frac{4 P_{e}^{s}\left(x_{1}^{n}\right)}{P_{e}^{0 s}\left(x_{1}^{n}\right) P_{e}^{1 s}\left(x_{1}^{n}\right)} \\
& =4 \exp \left\{n \cdot\left(\frac{1}{n} \log P_{e}^{s}\left(x_{1}^{n}\right)-\frac{1}{n} \log P_{e}^{0 s}\left(x_{1}^{n}\right) P_{e}^{1 s}\left(x_{1}^{n}\right)\right)\right\},
\end{aligned}
$$

where the inequality follows from applying (94) repeatedly. Due to the log sum inequality, we have

$$
\frac{1}{n} \log P_{e}^{s}\left(x_{1}^{n}\right)-\frac{1}{n} \log P_{e}^{0 s}\left(x_{1}^{n}\right) P_{e}^{1 s}\left(x_{1}^{n}\right) \rightarrow c_{s}<0,
$$

almost surely as $n \rightarrow \infty$, where $c_{s}$ is a negative constant since $s$ is not a state, and its children nodes must have different statistics. Using (113) in the bound (112) we obtain (111).

\section{EXPERIMENTAL RESULTS}

In Figure 10, we compare our BWT-based algorithm with the LZ string-matching based algorithm [15] as well as the zipping method [1]. The sources we use are randomly generated binary tree sources, with memory length 11, and 15 states. All the curves plotted are an average of 100 runs. As shown in Figure 10, 
the new algorithm converges quite fast to the true divergence. In contrast, for the data sizes considered, neither the LZ based algorithm nor the zipping method are able to offer good estimates. Although not shown in Figure 10, the estimate of the zipping method exhibits high sensitivity to the actual source realization.

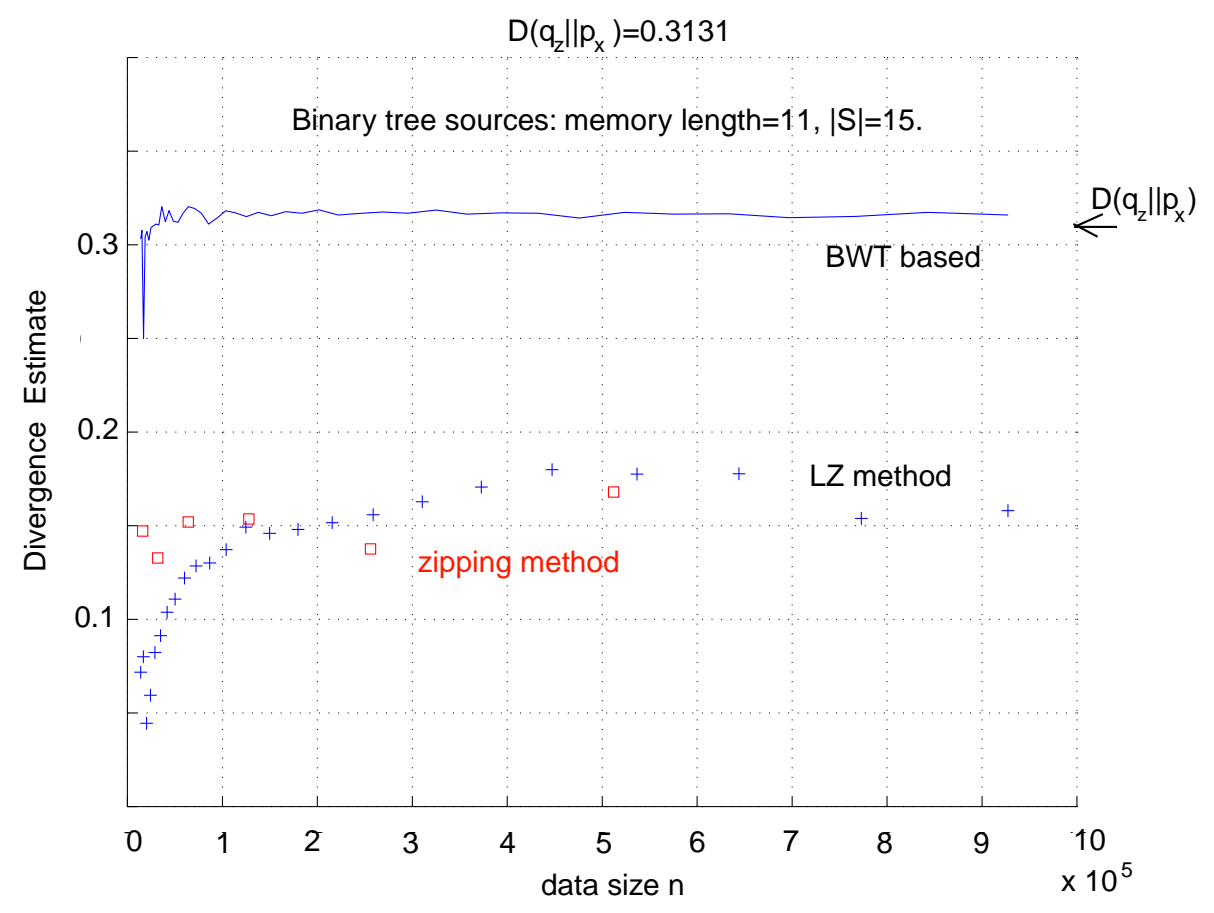

Fig. 10. Comparison of the methods in [1] (zipping) and [15] (LZ) with the new BWT-based method.

In Figure 11, we compare our BWT-based algorithm with the empirical plug-in scheme that assumes a Markov model of a given order. Even when the memory length is known the plug-in scheme does not perform as well as our scheme, and moreover, it suffers considerable degradation if it either underestimates or overestimates the order. Figure 11 also shows that the performance difference for adaptive and uniform segmentation is negligible.

In the empirical plug-in scheme, unless prior knowledge is available about the tree structure of the sources, the number of transition probabilities to be estimated grows exponentially with the assumed order of the source. Our algorithm has the advantage that it does not require any knowledge of the 


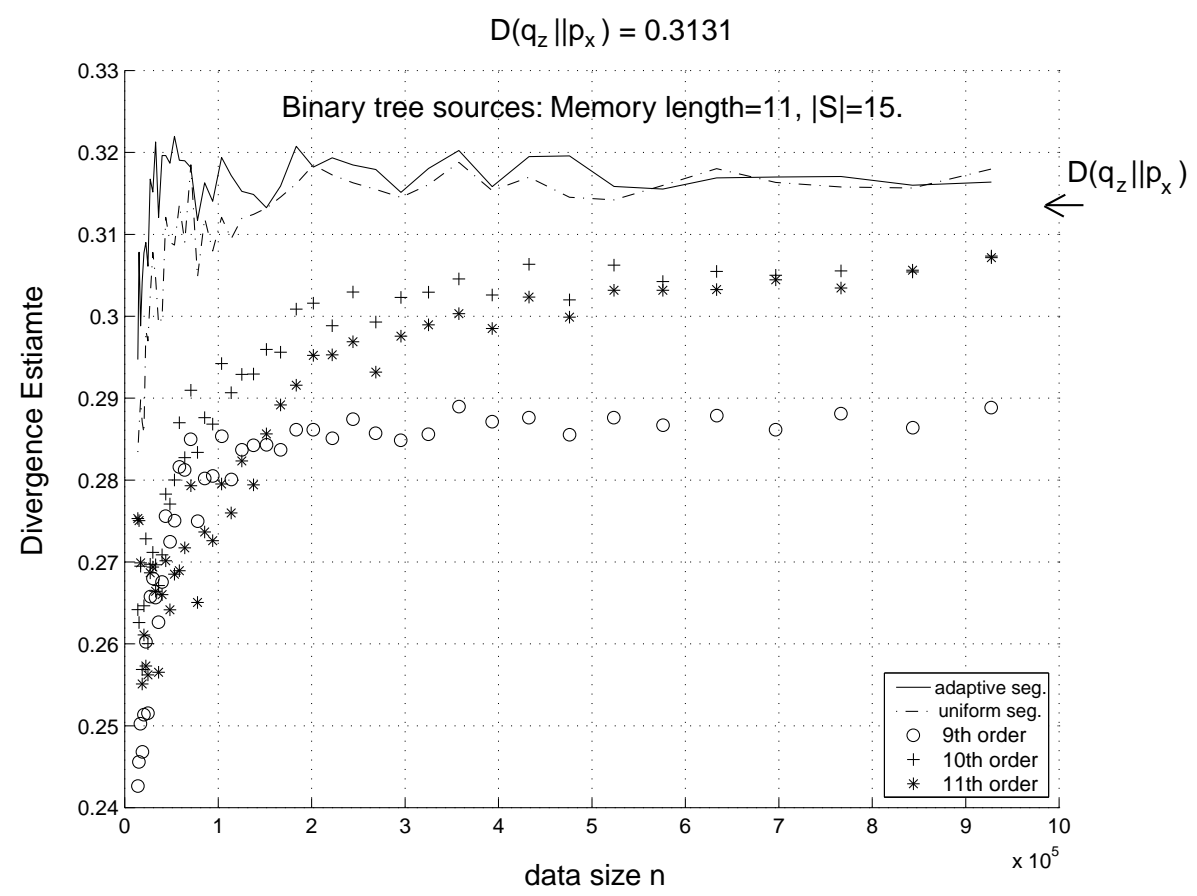

Fig. 11. Divergence estimator via the BWT and the plug-in estimator.

memory length or the number of states. Hence, it is suitable for unknown tree sources.

In Figure 12, we consider the effect of dependence between the realizations $\mathbf{x}$ and $\mathbf{z}$ on the divergence estimate. The sources tested in this figure have the same distributions as the sources in Figure 10. In particular, two extreme cases are tested. In the first case, sequence $\mathbf{x}$ and sequence $\mathbf{z}$ are independently generated. In the second case, $\mathbf{z}$ is generated by complementing $\mathbf{x}$. Since we chose the sources $p_{x}$ and $q_{z}$ to have mirror trees, the sequence $\mathbf{z}$ so generated does indeed have distribution $q_{z}$. The experiment shows that our divergence estimator is rather insensitive to the joint distribution, except through the marginals.

In Figures 13 and 14, we compare the BWT-based algorithm and the basic CTW-based algorithm (with $D=16$ ) for the same sources tested in Figure 10. While the divergence estimator via CTW exhibits slightly faster convergence, the divergence estimator via the BWT is easier to implement.

Our divergence estimator can be used in linguistic problems such as language analysis and author recognition. First the divergence estimator is used to classify texts written in different languages. The text files we use are the Bible (Old Testament) translated into 11 languages. The divergence table (based on the estimates via the BWT) of each pair of languages is shown Figure 15. (The divergence estimator via CTW gives similar estimates generally within 10\%.) Then we map the symmetrized distance matrix to a language tree using the Fitch-Margoliash method in the package PhylIP [8] for inferring evolutionary trees. Our algorithm successfully recognizes major language groups, such as Romance and Germanic (see 


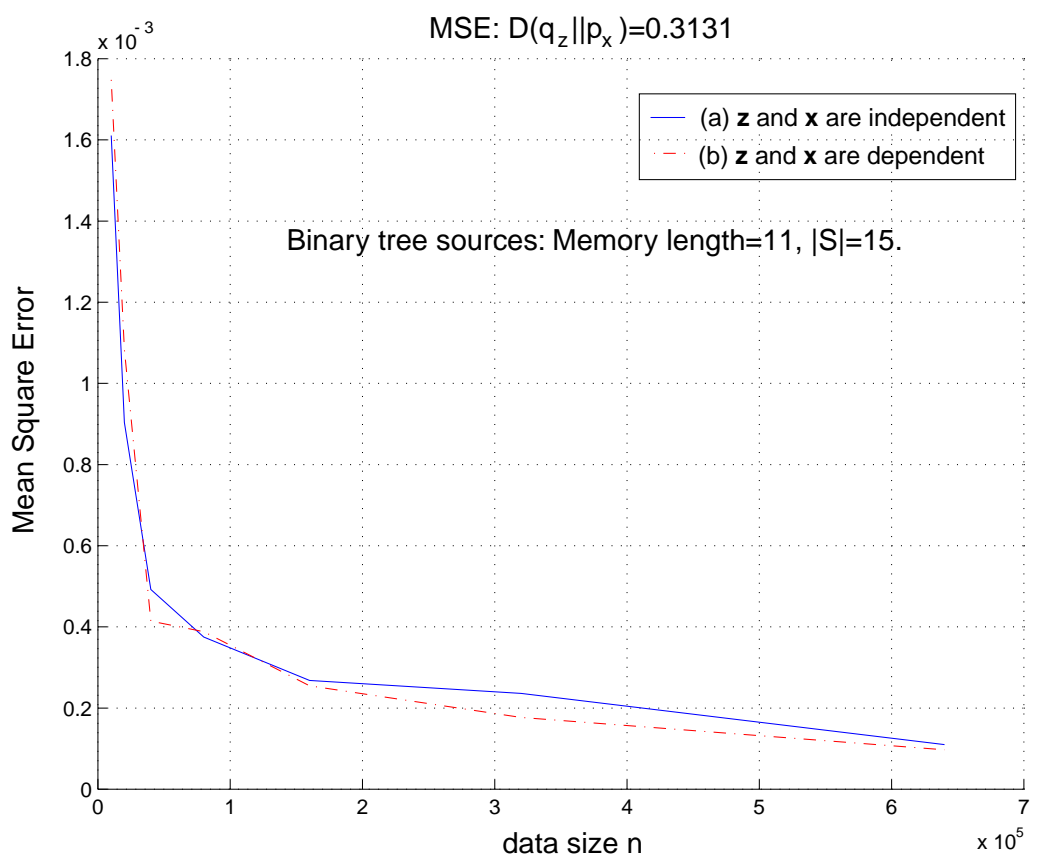

Fig. 12. Mean square error of the BWT-based divergence estimator with uniform segmentation: (a) $\mathbf{z}$ and $\mathbf{x}$ are independent realizations. (b) $\mathbf{z}$ is obtained by complementing $\mathbf{x}$. The sources generating $\mathbf{z}$ have the identical distributions in (a) and (b).

Figure 16). Note that according to our algorithm, English is a Germanic language, whereas according to the algorithm in [1], English is closer to the group of Romance languages. The experimental results of [1] were based on "The Universal Declaration of Human Rights" in 50 different languages.

We have also used the divergence estimator for discriminating between authors. We run the divergence estimator on nine English novels (or English translations). One of them is "Gadsby", a unique novel without a single letter ' $\mathrm{e}$ ' written by Ernest Vincent Wright in 1939. As shown in Figure 17, most divergence estimates are in the range of 0.15 to 0.34 , except $\bar{D}(G a d s b y|| \cdot)$ which are between 0.6 and 0.8 , and $\bar{D}(\cdot \| G a d s b y)$ which are between 1.2 and 1.4. In fact, $D(\cdot \| G a d s b y)$ should be infinity, because "Gadsby" does not have a single 'e', but the letter 'e' has a frequency of about $10 \%$ in English (and in particular has non-zero frequency in the other eight novels). However, in the case of infinite divergence, our estimator will not output infinity because we use a bias $\Delta>0$ in the probability estimates. But the divergence estimates will grow roughly at the rate of $C * \log _{2} \frac{\sqrt{n}}{\Delta}$. So either increasing the sequence length $n$ or decreasing $\Delta$ will cause the estimates to increase without bound. In contrast, note that when divergence is finite, the estimate is insensitive to shrinkage of $\Delta$.

Reassuringly, novels by the same author have relatively small divergence, such as "Anna Karenina" and "War and Peace" by Leo Tolstoy; and the four novels by Jane Austen. Interestingly, the divergence is also comparatively small for the novels written by the Bronte sisters: ("Jane Eyre" by Charlotte Bronte 


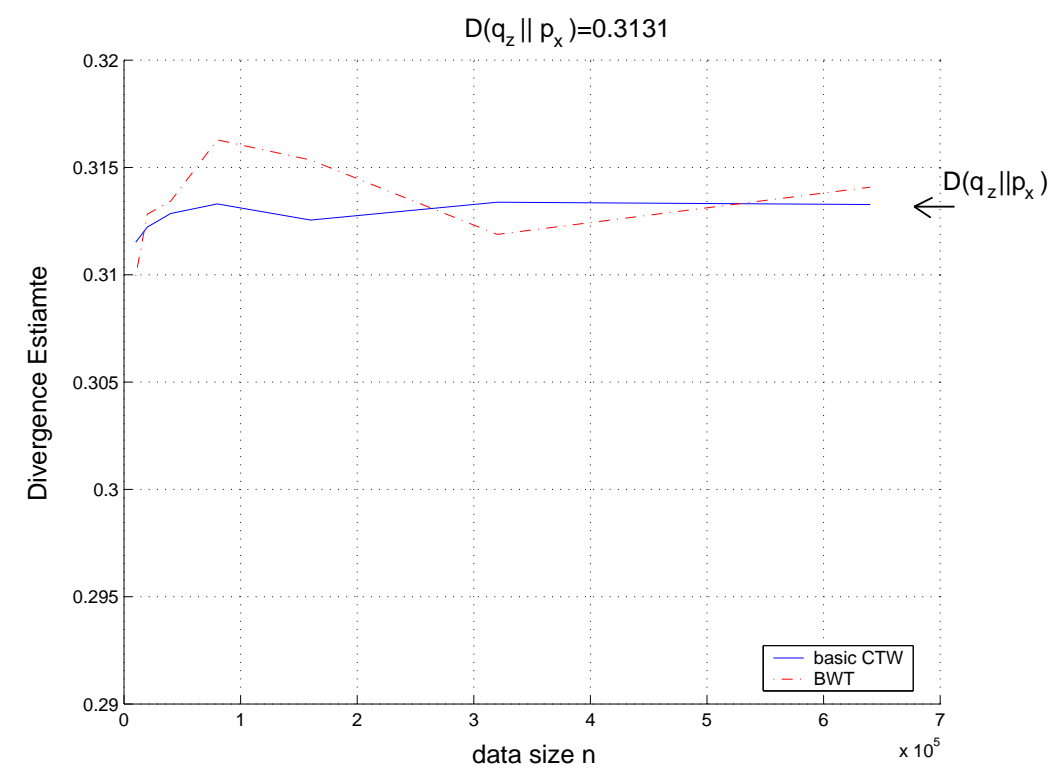

Fig. 13. Divergence estimator based on the BWT/CTW: $D\left(q_{z} \| p_{x}\right)=0.3131$.

and "Wuthering Heights" by Emily Bronte).

\section{APPENDIX A}

Let $f=p_{1}^{2}+p_{2}^{2}+p_{3}^{2}$. It follows from the two constraints (26), (27) that

$$
d p_{1}+d p_{2}+d p_{3}=0
$$

and

$$
\left(1+\log p_{1}\right) d p_{1}+\left(1+\log p_{2}\right) d p_{2}+\left(1+\log p_{3}\right) d p_{3}=0
$$

Therefore,

$$
\begin{aligned}
d f= & 2 p_{1} d p_{1}+2 p_{2} d p_{2}+2 p_{3} d p_{3} \\
= & 2\left(p_{1} \log p_{2}-p_{1} \log p_{3}+p_{2} \log p_{3}-p_{2} \log p_{1}\right. \\
& \left.+p_{3} \log p_{1}-p_{3} \log p_{2}\right) d p_{1} /\left(\log p_{2}-\log p_{3}\right) .
\end{aligned}
$$

When $p_{1}>p_{2}>p_{3}$, we shall prove $\frac{d f}{d p_{1}}>0$ in the following. We have

$$
\frac{d f}{d p_{1}}=\frac{2 g\left(p_{1}, p_{2}, p_{3}\right)}{\log p_{2}-\log p_{3}}
$$

where

$$
\begin{aligned}
g\left(p_{1}, p_{2}, p_{3}\right) & =p_{1} \log p_{2}-p_{1} \log p_{3}+p_{2} \log p_{3} \\
& -p_{2} \log p_{1}+p_{3} \log p_{1}-p_{3} \log p_{2} .
\end{aligned}
$$




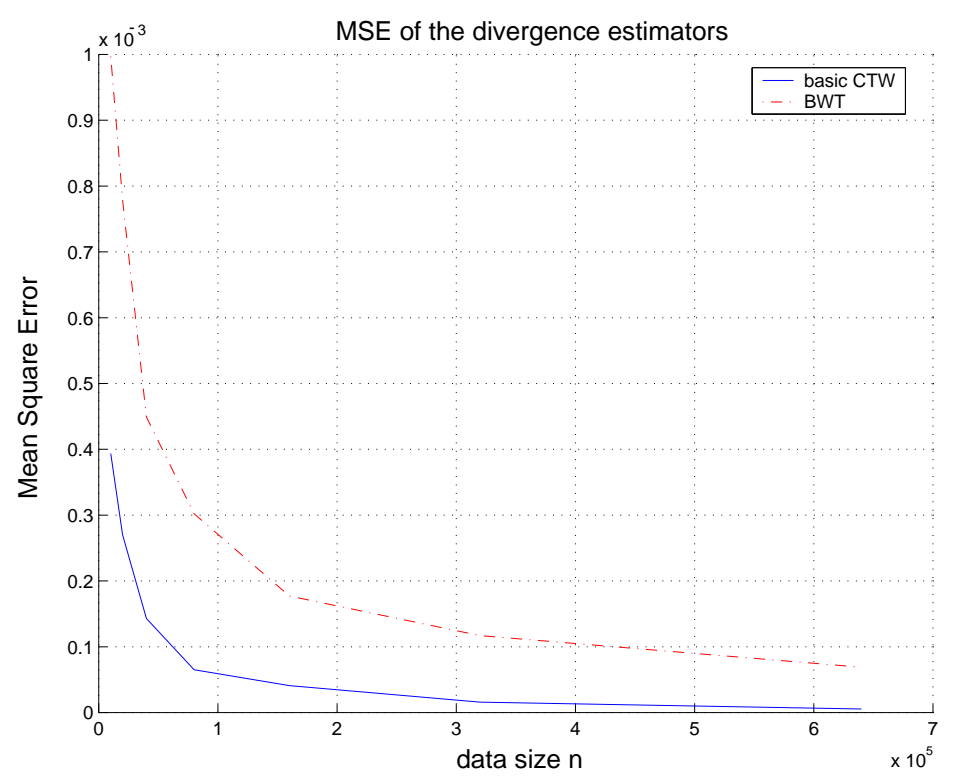

Fig. 14. Mean square error of the divergence estimator based on the BWT/CTW: $D\left(q_{z} \| p_{x}\right)=0.3131$.

\begin{tabular}{|c|c|c|c|c|c|c|c|c|c|c|c|}
\hline $\mathrm{D}(\mathrm{z} \| \mathrm{x}) \backslash \mathrm{x}$ & SPA & POR & FRE & ITA & ENG & GER & DUT & SWE & DAN & NOR & ICE \\
\hline $\mathrm{z}^{\text {Spanish }}$ & $/$ & 1.58 & 1.98 & 2.14 & 2.73 & 3.12 & 3.01 & 2.77 & 2.70 & 2.71 & 3.08 \\
\hline Portuguese & 1.40 & $/$ & 2.11 & 2.02 & 2.79 & 3.11 & 3.02 & 2.80 & 2.68 & 2.70 & 2.92 \\
\hline French & 2.17 & 2.34 & $/$ & 2.34 & 2.34 & 2.77 & 2.76 & 2.73 & 2.50 & 2.45 & 2.93 \\
\hline Italian & 1.85 & 1.94 & 2.16 & $/$ & 2.65 & 3.23 & 3.34 & 2.90 & 2.81 & 2.84 & 3.02 \\
\hline English & 3.01 & 3.49 & 2.75 & 3.24 & $/$ & 2.62 & 2.91 & 2.94 & 2.73 & 2.73 & 3.23 \\
\hline German & 2.95 & 3.46 & 2.66 & 3.09 & 2.50 & $/$ & 2.05 & 2.42 & 2.45 & 2.49 & 2.77 \\
\hline Dutch & 2.99 & 3.64 & 2.85 & 3.37 & 2.67 & 2.08 & $/$ & 2.49 & 2.31 & 2.55 & 3.20 \\
\hline Swedish & 2.79 & 3.13 & 2.64 & 2.82 & 2.34 & 2.11 & 2.26 & $/$ & 1.48 & 1.20 & 1.97 \\
\hline Danish & 2.77 & 3.01 & 2.36 & 2.73 & 2.29 & 1.95 & 2.03 & 1.10 & $/$ & 0.49 & 1.81 \\
\hline Norwegian & 2.85 & 3.16 & 2.41 & 2.77 & 2.36 & 2.06 & 2.14 & 0.99 & 0.45 & $/$ & 1.63 \\
\hline Icelandic & 2.95 & 3.19 & 2.81 & 2.85 & 2.90 & 2.50 & 2.80 & 1.97 & 1.99 & 1.79 & $/$ \\
\hline
\end{tabular}

Fig. 15. Divergence between languages (Bible) estimated by the BWT-based method.

We have $g\left(p_{1}, p_{2}, p_{3}\right)=0$ when $p_{1}=p_{2}$. Let

$$
h\left(p_{1}, p_{2}, p_{3}\right) \triangleq \frac{\partial g\left(p_{1}, p_{2}, p_{3}\right)}{\partial p_{1}}=\log p_{2}-\log p_{3}-\frac{p_{2}}{p_{1}}+\frac{p_{3}}{p_{1}} .
$$

We have $h\left(p_{1}, p_{2}, p_{3}\right)=0$ when $p_{2}=p_{3}$, and

$$
\frac{\partial h\left(p_{1}, p_{2}, p_{3}\right)}{\partial p_{2}}=\frac{1}{p_{2}}-\frac{1}{p_{1}}>0 .
$$

Therefore $h\left(p_{1}, p_{2}, p_{3}\right)>0$ and $g\left(p_{1}, p_{2}, p_{3}\right)>0$ if $p_{1}>p_{2}>p_{3}$. Hence, $f$ is an increasing function of $p_{1}$, and is maximized when either $p_{1}=1$ or $p_{1}>p_{2}=p_{3}$. 


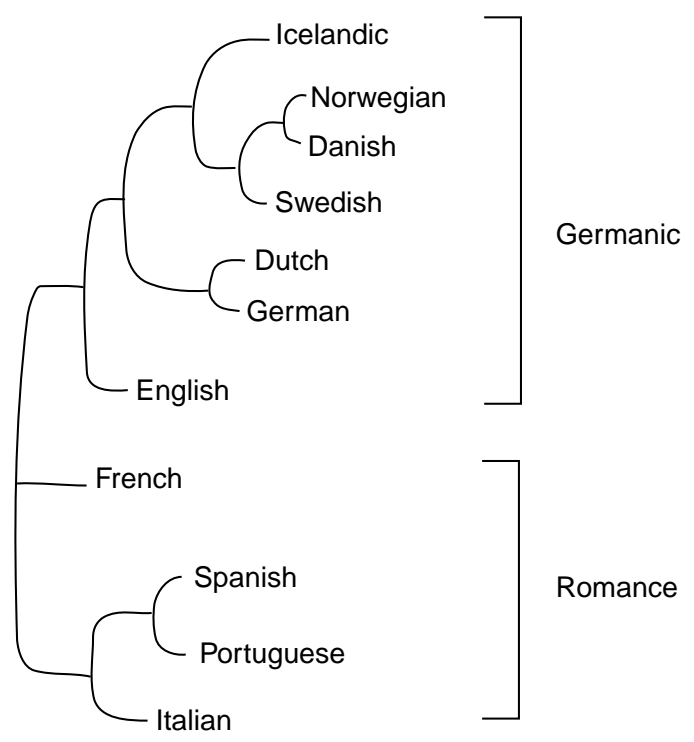

Fig. 16. Language tree derived from the divergence esimtates of Figure 15.

\begin{tabular}{|c|c|c|c|c|c|c|c|c|c|c|}
\hline \multicolumn{2}{|c|}{ Author $z^{\mathrm{D}(\mathrm{q} \| \mathrm{p})} \mathrm{P}^{\mathrm{x}}$} & \multirow{2}{*}{\begin{tabular}{|c|} 
Gadsby \\
1
\end{tabular}} & \multirow{2}{*}{\begin{tabular}{l|} 
Anna \\
0.69
\end{tabular}} & \multirow{2}{*}{\begin{tabular}{|l|} 
War \\
0.65 \\
\end{tabular}} & \multirow{2}{*}{$\begin{array}{l}\text { Jane } \\
0.61\end{array}$} & \multirow{2}{*}{$\begin{array}{l}\begin{array}{l}\text { Wuth- } \\
\text { ering }\end{array} \\
0.65\end{array}$} & \multirow{2}{*}{\begin{tabular}{|l|l|} 
Pride \\
0.78
\end{tabular}} & \multirow{2}{*}{$\begin{array}{l}\text { Mans- } \\
\text { field } \\
0.72\end{array}$} & \multirow{2}{*}{\begin{tabular}{|c|} 
Sense \\
0.76 \\
\end{tabular}} & \multirow{2}{*}{$\begin{array}{l}\text { Emma } \\
0.76\end{array}$} \\
\hline E. Wright & Gadsby (1939) & & & & & & & & & \\
\hline \multirow{2}{*}{ L. Tolstoy } & Anna K & 1.31 & I & 0.18 & 0.26 & 0.23 & 0.31 & 0.29 & 0.32 & 0.30 \\
\hline & War anc & 1.34 & 0.20 & 1 & 0.27 & 0.26 & 0.34 & 0.32 & 0.34 & 0.34 \\
\hline C. Bror & Jane E & 1.26 & 0.22 & 0.20 & I & 0.13 & 0.24 & 0.20 & 0.23 & 0.23 \\
\hline E. Bronte & Wuthering Heights (1847) & 1.32 & 0.23 & 0.22 & 0.16 & I & 0.26 & 0.24 & 0.25 & 0.26 \\
\hline \multirow{4}{*}{ J. Austen } & Pride and Prejudice (1813) & 1.45 & 0.30 & 0.29 & 0.25 & 0.24 & 1 & 0.18 & 0.19 & 0.19 \\
\hline & Mansfield Park (1814) & 1.32 & 0.26 & 0.25 & 0.20 & 0.21 & 0.17 & 1 & 0.17 & 0.16 \\
\hline & Sense \& Sensibility (1811) & 1.39 & 0.28 & 0.27 & 0.22 & 0.21 & 0.16 & 0.15 & I & 0.17 \\
\hline & Emma (1816) & 1.38 & 0.28 & 0.28 & 0.24 & 0.22 & 0.18 & 0.17 & 0.18 & 1 \\
\hline
\end{tabular}

Fig. 17. Divergence between novels estimated by the BWT-based method.

\section{APPENDIX B}

Let $\alpha=\alpha_{2}+\alpha_{3}$ and $\beta_{2}=\frac{\alpha_{2}}{\alpha_{2}+\alpha_{3}}$. We rewrite (28) as follows

$$
G_{3}(\overline{\bar{p}}, E)=\max _{p_{2}, p_{3}, \alpha, \beta_{2}}\left\{(1-\alpha)+\alpha\left(\beta_{2} p_{2}^{2}+\left(1-\beta_{2}\right) p_{3}^{2}\right)-\overline{\bar{p}}^{2}\right\}
$$

under the constraints

$$
\begin{gathered}
(1-\alpha)+\alpha\left(\beta_{2} p_{2}+\left(1-\beta_{2}\right) p_{3}\right)=\overline{\bar{p}} \\
\alpha\left(-\beta_{2} p_{2} \log p_{2}-\left(1-\beta_{2}\right) p_{3} \log p_{3}\right)=-\overline{\bar{p}} \log \overline{\bar{p}}-E,
\end{gathered}
$$

and $1 \geq \alpha \geq 0,1 \geq \beta_{2} \geq 0$, and $1 \geq p_{2} \geq 0,1 \geq p_{3} \geq 0$.

We first keep $\alpha$ fixed and maximize over $\beta_{2}, p_{2}$ and $p_{3}$. Define

$$
G_{2}\left(p^{\prime}, y^{\prime}\right) \triangleq \max _{p_{2}, p_{3}, \beta_{2}}\left\{\beta_{2} p_{2}^{2}+\left(1-\beta_{2}\right) p_{3}^{2}\right\}
$$


under the constraints

$$
\begin{gathered}
\beta_{2} p_{2}+\left(1-\beta_{2}\right) p_{3}=p^{\prime} \\
-\beta_{2} p_{2} \log p_{2}-\left(1-\beta_{2}\right) p_{3} \log p_{3}=y^{\prime}
\end{gathered}
$$

and $1 \geq \beta_{2} \geq 0,1 \geq p_{2} \geq 0,1 \geq p_{3} \geq 0$.

We can rewrite $G_{2}\left(p^{\prime}, y^{\prime}\right)=\max _{\theta} G\left(p^{\prime}, y^{\prime}, \theta\right)$ as an optimization problem over $\theta$. Notice that $G\left(p^{\prime}, y^{\prime}, \theta\right)=$ $\left(p_{2}+p_{3}\right) p^{\prime}-p_{2} p_{3}$, where $p_{2}$ and $p_{3}$ are determined by $p^{\prime}, y^{\prime}$, and $\theta$ through the constraints (see Figure 18). Since $p_{2}$ and $p_{3}$ are two intersections of the line $y=\tan \theta\left(x-p^{\prime}\right)+y^{\prime}$ and the curve $y=-x \log x$, they

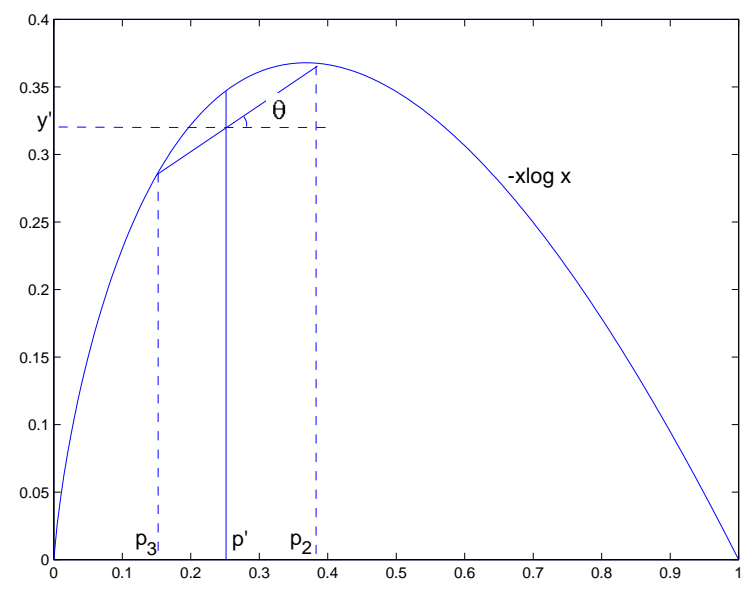

Fig. 18. Maximizing $G\left(p^{\prime}, y^{\prime}, \theta\right)=\left(p_{2}+p_{3}\right) p^{\prime}-p_{2} p_{3}$ over $\theta$, where $p_{2}$ and $p_{3}$ are two intersections of the line $y=\tan \theta\left(x-p^{\prime}\right)+y^{\prime}$ and the curve $y=-x \log x$.

are two solutions of the following equation:

$$
x \log x+\tan \theta\left(x-p^{\prime}\right)+y^{\prime}=0 .
$$

We have

$$
d p_{2}=-\frac{p_{2}-p^{\prime}}{\cos ^{2} \theta\left(1+\log p_{2}+\tan \theta\right)} d \theta
$$

and

$$
d p_{3}=-\frac{p_{3}-p^{\prime}}{\cos ^{2} \theta\left(1+\log p_{3}+\tan \theta\right)} d \theta
$$

Hence

$$
\begin{aligned}
\frac{d G}{d \theta} & =\left(p^{\prime}-p_{3}\right) \frac{d p_{2}}{d \theta}+\left(p^{\prime}-p_{2}\right) \frac{d p_{3}}{d \theta} \\
& =-\frac{\left(p_{2}-p^{\prime}\right)\left(p^{\prime}-p_{3}\right)}{\cos ^{2} \theta}\left(\frac{1}{\tan \theta+1+\log p_{2}}+\frac{1}{\tan \theta+1+\log p_{3}}\right)
\end{aligned}
$$


where $\tan \theta=\frac{-p_{2} \log p_{2}+p_{3} \log p_{3}}{p_{2}-p_{3}}$. Without loss of generality, we assume $p_{2}>p^{\prime}>p_{3}$. In Appendix $\mathrm{C}$, we prove that

$$
\begin{aligned}
& \tan \theta+1+\log p_{2}>0 \\
& \tan \theta+1+\log p_{3}<0
\end{aligned}
$$

and

$$
\tan \theta+1+\log p_{2}<-\left(\tan \theta+1+\log p_{3}\right) .
$$

From (130) (131) (132) and (133), we have

$$
\frac{d G}{d \theta}<0
$$

Therefore, the maximum $G_{2}\left(p^{\prime}, y^{\prime}\right)=\max _{\theta} G\left(p^{\prime}, y^{\prime}, \theta\right)$ is achieved when $\tan \theta=\frac{-y^{\prime}}{1-p^{\prime}}$ (in which case $p_{2}=1$ and $p_{3}<p^{\prime}$ satisfying $\left.p_{3} \log p_{3}+\frac{-y^{\prime}}{1-p^{\prime}}\left(p_{3}-p^{\prime}\right)+y^{\prime}=0\right)$. From the solution of the optimization problem (124), we see that (121) is maximized when $p_{2}=1$ (or equivalently $\beta_{2}=0$ ).

\section{APPENDIX C}

Under the assumption $p_{2}>p_{3}$, we want to prove the inequalities

$$
\begin{aligned}
& 1+\log p_{2}+\frac{-p_{2} \log p_{2}+p_{3} \log p_{3}}{p_{2}-p_{3}}>0 \\
& 1+\log p_{3}+\frac{-p_{2} \log p_{2}+p_{3} \log p_{3}}{p_{2}-p_{3}}<0
\end{aligned}
$$

and

$$
2+\log p_{2}+\log p_{3}+\frac{2\left(-p_{2} \log p_{2}+p_{3} \log p_{3}\right)}{p_{2}-p_{3}}<0
$$

We have

$$
\frac{p_{3}}{p_{2}-p_{3}}\left(\frac{p_{2}}{p_{3}}-1-\log \frac{p_{2}}{p_{3}}\right)>0
$$

and

$$
-\frac{p_{2}}{p_{2}-p_{3}}\left(\frac{p_{3}}{p_{2}}-1-\log \frac{p_{3}}{p_{2}}\right)<0
$$

since $p_{2}>p_{3}$ and $x-1-\log x>0$ holds for all $x>0$ and $x \neq 1$. Let

$$
f(x)=2\left(x-p_{3}\right)-\left(x+p_{3}\right)\left(\log x-\log p_{3}\right)
$$

we have $f\left(p_{3}\right)=0$ and for all $x>p_{3}$,

$$
f^{\prime}(x)=\log \frac{p_{3}}{x}+1-\frac{p_{3}}{x}<0 .
$$

Therefore, $f\left(p_{2}\right)<0$. So we have

$$
\frac{2\left(p_{2}-p_{3}\right)-\left(p_{2}+p_{3}\right)\left(\log p_{2}-\log p_{3}\right)}{p_{2}-p_{3}}<0 .
$$




\section{APPENDIX D}

In order to prove (38), we need to prove that

$$
E\left[\left(\frac{N_{\mathbf{z}}(a, s)}{n}-q_{z}(s) q_{z}(a \mid s)\right)^{2}\right]=O\left(\frac{1}{n}\right) .
$$

Notice that $S_{1}^{\prime}, S_{2}^{\prime}, \ldots$ form a Markov chain with the stationary distribution $q_{z}(a, s)=q_{z}(s) q_{z}(a \mid s)$, where $S_{i}^{\prime}=\left(S_{i}, Z_{i}\right), i=1,2, \ldots, n$.

$$
N_{\mathbf{z}}(a, s)=\sum_{i=1}^{n} \mathbf{1}_{\left\{S_{i}=s, Z_{i}=a\right\}}=\sum_{i=1}^{n} \mathbf{1}_{\left\{S_{i}^{\prime}=(s, a)\right\}} .
$$

Then, (143) follows from the fact [4] that

$$
E\left[\left(\frac{N_{\mathbf{z}}\left(s^{\prime}\right)}{n}-q_{z}\left(s^{\prime}\right)\right)^{2}\right]=O\left(\frac{1}{n}\right) .
$$

\section{APPENDIX E}

Under the assumption that the Markov chain is irreducible and aperiodic, there exists an integer $k_{0}=$ $d_{0}+c_{0}$, where $d_{0}$ is the order of the Markov source and $c_{0} \geq 0$ is a constant, such that

$$
\max _{s_{i}, s_{j} \in \mathcal{S}, A \subset \mathcal{S}}\left|p_{x}^{\left(k_{0}\right)}\left(s_{i}, A\right)-p_{x}^{\left(k_{0}\right)}\left(s_{j}, A\right)\right|=\zeta<1,
$$

where $p_{x}^{\left(k_{0}\right)}\left(s_{i}, A\right)$ is the $k_{0}$-step transition probability. It follows that (see [5],[12]) for any $s \in \mathcal{S}$ and any $A \subset \mathcal{S}$,

$$
\left|p_{x}^{(n)}(s, A)-\mu_{x}(A)\right| \leq \zeta^{\left(n / k_{0}\right)-1}=\gamma_{0} \rho^{n}
$$

where $\rho=\zeta^{1 / k_{0}}$ and $\gamma_{0}=\zeta^{-1}$.

For arbitrary initial states $s_{i}$ and $s_{j}$, we have

$$
\max _{A \subset \chi^{l}}\left|p_{x}^{\left(l+c_{0}\right)}\left(s_{i}, A\right)-p_{x}^{\left(l+c_{0}\right)}\left(s_{j}, A\right)\right|=\max _{A \subset \mathcal{S}}\left|p_{x}^{\left(d_{0}+c_{0}\right)}\left(s_{i}, A\right)-p_{x}^{\left(d_{0}+c_{0}\right)}\left(s_{j}, A\right)\right|,
$$

where $l \geq d_{0}$. It follows that

$$
\max _{s_{i}^{\prime}, s_{j}^{\prime} \in \chi^{l}, A \subset \chi^{l}}\left|p_{x}^{\left(l+c_{0}\right)}\left(s_{i}^{\prime}, A\right)-p_{x}^{\left(l+c_{0}\right)}\left(s_{j}^{\prime}, A\right)\right|=\zeta<1 .
$$

Therefore, for any $s^{\prime} \in \chi^{l}$ and any $A^{\prime} \subset \chi^{l}$, we have

$$
\left|p_{x}^{(n)}\left(s^{\prime}, A^{\prime}\right)-\mu_{x}\left(A^{\prime}\right)\right| \leq \zeta^{\frac{n}{l+c_{0}}-1}=\gamma_{0} \rho_{l}^{n},
$$

where $\rho_{l}=\zeta^{\frac{1}{l+c_{0}}}$ and $\gamma_{0}=\zeta^{-1}$. 


\section{APPENDIX F}

Lemma 5 Suppose $\mathrm{x}$ is a sequence of length $n$ generated from Markov source $p_{x}$ with alphabet $\chi$ and order $d_{0}$. The Markov chain is assumed to be irreducible and aperiodic. Let $W$ be a set of nodes in the context tree that have depths no more than $l=o(n)$. Assume $W$ is equivalent to a set of consecutive nodes at depth $l$ in the context tree. Let $N_{\mathbf{x}}(W)$ be the number of symbols in the sequence $\mathbf{x}$ that have a context in $W$ and $\mu_{x}(W)$ be the stationary probability of $W$. Then we have

$$
E\left[\left(\frac{1}{n} N_{\mathbf{x}}(W)-\mu_{x}(W)\right)^{2}\right]=O\left(\frac{l^{3}}{n}\right) .
$$

Proof: We first find the upper bound of the number of nodes in $W$, where the children nodes in $W$ are replaced by the parent node, if all children nodes of a node (of depth at least $d_{0}$ ) are included in $W$. In addition, $W$ is assumed to consist of consecutive nodes. Thus, the number of nodes in $W$ is upper bounded by $2|\chi| l+|\chi|^{d_{0}}$, since for each depth larger than $d_{0}$ there are at most $2|\chi|$ nodes included in $W$. Therefore, we have

$$
E\left[\left(\frac{1}{n} N_{\mathbf{x}}(W)-\mu_{x}(W)\right)^{2}\right] \leq\left(2|\chi| l+|\chi|^{d_{0}}\right)^{2} \max _{s \in \cup_{d_{0} \leq m \leq l} \chi^{m}} E\left[\left(\frac{1}{n} N_{\mathbf{x}}(s)-\mu_{x}(s)\right)^{2}\right] .
$$

Now suppose $s$ is any node of depth $m, d_{0} \leq m \leq l$. We have

$$
E\left[\left(\frac{1}{n} N_{\mathbf{x}}(s)-\mu_{x}(s)\right)^{2}\right]=\frac{\mu_{x}(s)-\mu_{x}^{2}(s)}{n}+\frac{2 \mu_{x}(s)}{n^{2}} \sum_{d=1}^{n-1}(n-d)\left(p_{x}^{(d)}(s, s)-\mu_{x}(s)\right) .
$$

It follows from (150) in Appendix E that

$$
\begin{aligned}
\sum_{d=1}^{\infty}\left|p_{x}^{(d)}(s, s)-\mu_{x}(s)\right| & \leq \sum_{d=1}^{\infty} \zeta^{\frac{d}{m+c_{0}}-1} \\
& =\frac{1}{\zeta\left(1-\zeta^{\frac{1}{m+c_{0}}}\right)}-\frac{1}{\zeta} \\
& \leq \frac{1}{\zeta\left(1-\zeta^{\frac{1}{l+c_{0}}}\right)} \\
& =O\left(\frac{l+c_{0}}{\zeta \log \frac{1}{\zeta}}\right)
\end{aligned}
$$


and

$$
\begin{aligned}
\sum_{d=1}^{\infty} d\left|p_{x}^{(d)}(s, s)-\mu_{x}(s)\right| & \leq \sum_{d=1}^{\infty} d \zeta^{\frac{d}{m+c_{0}}-1} \\
& =\frac{\zeta^{\frac{1}{m+c_{0}}}}{\zeta\left(1-\zeta^{\frac{1}{m+c_{0}}}\right)^{2}} \\
& \leq \frac{1}{\zeta\left(1-\zeta^{\frac{1}{l+c_{0}}}\right)^{2}} \\
& =O\left(\frac{\left(l+c_{0}\right)^{2}}{\zeta \log ^{2} \frac{1}{\zeta}}\right)
\end{aligned}
$$

Combining (153) (157) and (161), we have

$$
E\left[\left(\frac{1}{n} N_{\mathbf{x}}(s)-\mu_{x}(s)\right)^{2}\right]=O\left(\frac{l}{n}+\frac{l^{2}}{n^{2}}\right) .
$$

From (152) and (162), we obtain (151) where $l=o(n)$.

\section{REFERENCES}

[1] D. Benedetto, E. Caglioti, V. Loreto. "Language trees and zipping". Physical Review Letters, Vol. 88, No. 4, 28 Jan, 2002.

[2] M. Burrows, D. J. Wheeler. "A block-sorting lossless data compression algorithm”. Tech. Rep. 124, Digital Systems Research Center, 1994.

[3] H. Cai, S. Kulkarni, S.Verdú. "Universal estimation of entropy and divergence via block sorting”. Proc. on IEEE ISIT 2002, pp 433, Lausanne, Switzerland, July 2002.

[4] H. Cai, S. Kulkarni, S. Verdú. "Universal entropy estimation via block sorting”. IEEE Trans. Inform. Theory, Vol. 50, No. 7, pp 15511561, July 2004.

[5] J. Doob. "Stochastic processes". New York: Wiley, 1953.

[6] J. G. Cleary, I. H. Witten. "Data compression using adaptive coding and partial string matching". IEEE Trans. Commun. Theory, Vol. 32, No. 4, pp 396-402, April 1984.

[7] Z. Dawy, J. Hagenauer, A. Hoffmann. "Implementing the Context Tree Weighting Method for Context Recognition”, DCC'04, Snowbird, Utah, March 2004.

[8] J. Felsenstein. Cladistics 5, pp 164-166, 1989.

[9] R. M. Gray. Entropy and Information Theory. Springer-Verlag, New York, 1990.

[10] A. Kaltchenko. "Algorithms for Estimation of Information Distance with Application to Bioinformatics and Linguistics", 2004 Canadian Conference on Electrical and Computer Engineering Niagara Fall, May 2004.

[11] M. Li, X. Chen, X. Li, B. Ma, P. Vitanyi. "The similarity metric". Proc. 14th ACM-SIAM SODA, 2003.

[12] S. V. Nagaev. "More exact statements of limit theorems for homogeneous markov chains". Theory Prob. Appl. Vol. 6, pp 62-81, 1961.

[13] F. M. J. Willems, Y. M. Shtarkov, T. J. Tjalkens. "The context tree weighting method : basic properties", IEEE Trans. Inform. Theory, Vol. 41, pp 653-664, May 1995.

[14] F. M. J. Willems. "The Context Tree Weighting Method: Extensions”, IEEE Trans. on Inform. Theory, Vol. 44, No. 2, pp. 792-798, March 1998.

[15] J. Ziv, N. Merhav. "A measure of relative entropy between individual sequences with application to universal classification". IEEE Trans. Inform. Theory, Vol. 39, No. 4, pp 1270-1279, July 1993. 